University of Wollongong

Research Online

Faculty of Engineering and Information

Faculty of Engineering and Information

Sciences - Papers: Part B

Sciences

2019

Optimal investment and consumption under a continuous-time cointegration model with exponential utility

Guiyuan Ma

University of Wollongong, guiyuan@uow.edu.au

Song-Ping Zhu

University of Wollongong, spz@uow.edu.au

Follow this and additional works at: https://ro.uow.edu.au/eispapers1

Part of the Engineering Commons, and the Science and Technology Studies Commons

Research Online is the open access institutional repository for the University of Wollongong. For further information contact the UOW Library: research-pubs@uow.edu.au 


\title{
Optimal investment and consumption under a continuous-time cointegration model with exponential utility
}

\author{
Abstract \\ In this paper, we study the effects of cointegration on optimal investment and consumption strategies for \\ an investor with exponential utility. A Hamilton-Jacobi-Bellman (HJB) equation is derived first and then \\ solved analytically. Both the optimal investment and consumption strategies are expressed in closed \\ form. A verification theorem is also established to demonstrate that the solution of the HJB equation is \\ indeed the solution of the original optimization problem under an integrability condition. In addition, a \\ simple and sufficient condition is proposed to ensure that the integrability condition is satisfied. \\ Financially, the optimal investment and consumption strategies are decomposed into two parts: the \\ myopic part and the hedging demand caused by cointegration. Discussions on the hedging demand are \\ carried out first, based on analytical formulae. Then numerical results show that ignoring the information \\ about cointegration results in a utility loss.

\section{Disciplines \\ Engineering | Science and Technology Studies}

\section{Publication Details}

Ma, G. \& Zhu, S. (2019). Optimal investment and consumption under a continuous-time cointegration model with exponential utility. Quantitative Finance, Online First 1-15. 


\title{
Optimal investment and consumption under a continuous-time cointegration model with exponential utility
}

\author{
Guiyuan $\mathrm{Ma}^{\dagger} \quad$ Song-Ping $\mathrm{Zhu}^{\ddagger}$
}

\begin{abstract}
In this paper, we study the effects of cointegration on optimal investment and consumption strategies for an investor with exponential utility. A Hamilton-Jacobi-Bellman (HJB) equation is derived first and then solved analytically. Both the optimal investment and consumption strategies are expressed in closed form. A verification theorem is also established to demonstrate that the solution of the HJB equation is indeed the solution of the original optimization problem under an integrability condition. In addition, a simple and sufficient condition is proposed to ensure that the integrability condition is satisfied. Financially, the optimal investment and consumption strategies are decomposed into two parts: the myopic part and the hedging demand caused by cointegration. Discussions on the hedging demand are carried out first, based on the analytical formula. Then numerical results show that ignoring the information about cointegration results in a utility loss.
\end{abstract}

Keywords. Cointegration; Hamilton-Jacobi-Bellman (HJB) equation; Optimal investment and consumption problem; Closed-form analytical solutions; Verification theorem.

\footnotetext{
${ }^{\dagger}$ Corresponding author. School of Mathematics and Applied Statistics, University of Wollongong, NSW 2522, Australia. Email: guiyuan@uow.edu.au.

${ }^{\ddagger}$ School of Mathematics and Applied Statistics, University of Wollongong, NSW 2522, Australia. Email: spz@uow.edu.au.
} 


\section{Introduction}

Portfolio selection is one of the most important topics in mathematical finance. Its pioneering work was attributed to Markowitz (1952), who established an elegant mathematical framework for optimal portfolio selection problem in a single-period setting. Later, Samuelson (1969) and Merton $(1969,1971)$ extended Markowitz's work in a multi-period setting and a continuous-time setting, respectively. In these aforementioned works, they all assumed that the asset returns are independent and identically distributed (i.i.d.), which makes the corresponding strategies be myopic for the investors with mean-variance, constant-relative-risk-aversion (CRRA) or constantabsolute-risk-aversion (CARA) preferences.

Since the publication of these landmark papers, optimal portfolio selection problem has attracted very high attention in academia and many important extensions have been proposed. One important extension is to reconcile the non-stationarities of asset returns. From empirical studies, Campbell (1987), Campbell and Shiller (1988) and Fama and French (1988) found that asset returns are non-stationary, i.e. the i.i.d assumption is violated. To remedy this assumption, several stochastic models were developed from various financial aspects. Kim and Omberg (1996) assumed that the risk premium follows an Ornstein-Uhlenbeck (O-U) process. Brennan et al. (1997) also assumed that the risk premium is predictable by a stochastic interest rate and a dividend yield. Later, Wachter (2002) derived a closed-form solution for the continuous-time optimal investment and consumption problem with return predictability. Finally, Liu (2007) provided a general treatment of models under stochastic environments and presented the optimal allocation accordingly.

In this paper, we consider the optimal investment and consumption problem under a cointegration model. Cointegration was first introduced by Granger (1981), who discovered that a linear combination of two or more non-stationary time series could be stationary. Engle and Granger (1987) further developed some necessary statistical techniques for cointegration and a rigorous proof for Granger's representation theorem. Cointegration now has been successfully applied in various economic models and Granger was thus awarded the Nobel Prize in Economics in 2003 .

The cointegration phenomena have been observed from different real markets. Cerchi and 
Havenner (1988) first found stock prices were cointegrated from empirical data. Later, Baillie and Bollerslev (1989), Taylor and Tonks (1989), and Serletis (1994) also observed the cointegration phenomena from financial data of exchange rate, market indices and energy prices, respectively. As a result of cointegration phenomena being observed in different financial markets, the concept of cointegration has been adopted gradually in various classic financial problems. Alexander (1999) demonstrated the application of cointegration in hedging and asset allocation. Duan and Pliska (2004) reconsidered option pricing when the underlying stock prices are cointegrated. Mudchanatongsuk et al. (2008) proposed a stochastic control approach to the problem of pair trading under cointegration via maximizing the power utility defined on the terminal wealth. Later, Tourin and Yan (2013) explored a similar problem under exponential utility and also provided a verification theorem. However, consumption is not taken into account in either Mudchanatongsuk et al. (2008) or Tourin and Yan (2013). Almost at the same time, Chiu and Wong (2011, 2012, 2013a) studied the continuous-time mean-variance portfolio selection problem in a complete market and an incomplete market under the assumption that the asset prices are cointegrated. Chiu and Wong (2013b) further studied the optimal investment problem for an insurer who invests in cointegrated assets. However, they did not take the consumption into consideration in their works, either. Until very recently, Shen and Siu (2017) solved the optimal investment and consumption problem under the cointegration model, in order to maximize the expected power or logarithmic utility obtained from both the terminal wealth and the intermediate consumption. In their paper, Shen and Siu (2017) applied the Girsanov's theorem without checking the Novikov condition when establishing the verification theorem. To the best of our knowledge, an optimal investment and consumption problem in a continuous-time cointegrated model with exponential utility has not been well explored in the literature as it is hard to establish a proper verification theorem.

The main contribution of this paper is that we examine the effects of cointegration on the optimal investment and consumption problem analytically for an investor with exponential utility. An HJB equation is derived first using dynamic programming method and then solved analytically. Both the optimal investment and consumption strategies are expressed in closed form. A proper verification theorem is also established under an exponential integrability condition. Furthermore, a simple and sufficient condition is also provided to ensure that the integrability condition is 
satisfied. Financially, the optimal investment and consumption strategies are decomposed into two parts: the myopic part (i.e. the Merton component) and the hedging demand (the adjustment term) caused by cointegration. Utilizing the newly-derived analytical formula, we are able to carry out some discussions on how the hedging demand changes as the mean-reverting speed of cointegrating factor increases. Under the current parameter setting, the numerical results indicate that the more pronounced the cointegration effect is, the more the expected utility is improved. In other words, ignoring the information about cointegration results in a utility loss. Furthermore, horizon effect is numerically shown that the longer investment horizon, the more the investor takes advantage of the cointegration properties of the stocks.

The rest of the paper is organized as follows. In Section 2, a cointegration model is presented first and then the optimal investment and consumption problem is reformulated as a stochastic optimal control problem. An HJB equation is then derived when utility function is of exponential form. In Section 3, an analytical solution to the HJB equation is obtained first and then a proper verification theorem is established with a simple and sufficient condition. In Section 4, some numerical results are provided based on the obtained optimal consumption and investment strategies. The final section concludes the paper.

\section{Problem formulation}

\subsection{Cointegration model}

Cointegration was first proposed by Granger (1981) when he discovered that a linear combination of two or more non-stationary time series could become stationary. The collection of these time series variable is said to be cointegrated. According to Granger's representation theorem (Engle and Granger, 1987), the cointegrated vector time series can be expressed as an error-correction model.

In a discrete-time vector autoregressive (VAR) model, an error-correction dynamic for $m$ - 
component asset price time series with $k$ cointegration factors is defined as follows:

$$
\begin{aligned}
& \ln S_{i, t}-\ln S_{i, t-1}=\mu+\sum_{j=1}^{k} \delta_{i, j} z_{j, t-1}+\sigma_{i, t} \xi_{i, t}, i=1, \cdots, m, \\
& z_{j, t}=a_{j}+b_{j} t+\sum_{i=1}^{m} c_{i j} \ln S_{i, t} \quad j=1, \cdots, k,
\end{aligned}
$$

where $S_{i, t}$ is the price of asset $i$ at time $t$ for $i=1, \cdots, m,\left(c_{1 j}, \cdots, c_{m j}\right)$ are linearly independent vectors for $j=1, \cdots, k$ and the random vector $\left(\xi_{1, t}, \cdots, \xi_{m, t}\right)$ follows a multivariate normal distribution with mean zero and a constant covariance matrix. In the error-correction model, the vector of $k$ cointegrating factors, $\left(z_{1, t}, \cdots, z_{k, t}\right)$, should be stationary, which implies that the log-return in (2.1) is stationary. Duan and Pliska (2004) derived a continuous-time model by taking the diffusion limit of the discrete-time error-correction model. To reflect the spirit of the error-correction model in its diffusion limit, Chiu and Wong (2011, 2012, 2013b) assumed that the cointegrating factor follows an Ornstein-Uhlenbeck process and thus proposed a continuous-time cointegration model. For more details about the continuous-time cointegration model, readers are referred to Duan and Pliska (2004); Chiu and Wong (2011, 2012, 2013b).

\subsection{The financial market}

Consider a financial market consisting of $m+1$ assets: one risk-free asset and $m$ risky assets. All of these assets, labelled as $S_{i}$ for $i=0,1,2, \cdots, m$, are assumed to be traded continuously within time horizon $T$. The price of the risk-free asset evolves over time as follows:

$$
d S_{0}(t)=r(t) S_{0}(t) d t
$$

where $r(t)$ is the risk-free rate, which is assumed to be a given deterministic function of time $t$ and bounded on $[0, T]$. To characterize the dynamics of the cointegrated asset prices, we denote the log-price of the risky assets as

$$
X_{i}(t)=\ln S_{i}(t), \quad i=1, \cdots, m .
$$


In the continuous-time cointegration model (Chiu and Wong, 2011), the vector of log-prices of risky assets, $\mathbf{X}(t)=\left(X_{1}(t), \cdots, X_{m}(t)\right)^{\top}$, satisfies the following stochastic differential equation $(\mathrm{SDE})$

$$
d \mathbf{X}(t)=[\boldsymbol{\theta}(t)-\mathbf{A} \mathbf{X}(t)] d t+\boldsymbol{\sigma}(t) d \mathbf{W}(t), \quad t \in[0, T]
$$

where $\{\mathbf{W}(t)\}_{0 \leq t \leq T}$ is an $m$-dimensional Brownian motion defined on a fixed filtered complete probability space $\left(\Omega, \mathcal{F}, \mathbf{P},\left\{\mathcal{F}_{t}\right\}_{0 \leq t \leq T}\right)$, In (2.5), $\mathbf{A}$ is an $m \times m$ constant coefficient matrix of cointegration and $\boldsymbol{\Sigma}(t) \triangleq \boldsymbol{\sigma}(t) \boldsymbol{\sigma}^{\top}(t)$ represents the covariance matrix. It is assumed that the non-degeneracy condition $\boldsymbol{\Sigma}(t) \geq \delta \mathbf{I}_{m}$ holds for all $t \in[0, T]$ and for some $\delta>0$. In addition, all $\boldsymbol{\theta}(t), \boldsymbol{\sigma}(t), \boldsymbol{\Sigma}(t)$ and $\boldsymbol{\Sigma}^{-1}(t)$ are assumed to be deterministic functions of time $t$ and bounded on $[0, T]$. Hereafter, all notations denoted in boldfaced symbols are vectors or matrices; while those denoted by non-boldfaced symbols represent scalars.

Denote $\mathbf{S}(t)=\left(S_{1}(t), \cdots, S_{m}(t)\right)^{\top}$. Under the continuous-time cointegration model in (2.5), the dynamics of asset prices can be derived by using the Ito's formula as follows:

$$
d \mathbf{S}(t)=\operatorname{diag}(\mathbf{S}(t))[\boldsymbol{\alpha}(t) d t+\boldsymbol{\sigma}(t) d \mathbf{W}(t)], \quad t \in[0, T],
$$

where $\boldsymbol{\alpha}(t)=\boldsymbol{\theta}(t)-\mathbf{A} \mathbf{X}(t)+\frac{1}{2} \mathcal{D}(\boldsymbol{\Sigma}(t)) \mathbf{1}_{m \times 1}, \mathcal{D}(\boldsymbol{\Sigma})$ is a diagonal matrix with all diagonal elements equal to those of $\boldsymbol{\Sigma}$, and $\mathbf{1}_{m \times 1}$ is an $m \times 1$ unit vector.

\subsection{Optimal investment and consumption problem}

Consider an investor with a known initial wealth $y_{0}>0$. At any time $t$, prior to $T$, the investor needs to make a decision on how much to be consumed and, in the mean time, how much to be invested in the risky asset, in order to maximize his expected utility from the intermediate consumption and the terminal wealth. Let $u_{i}(t)$ be the investment amount on the $i$-th risky asset at time $t$ and $c(t)$ denote the consumption rate at time $t . \mathbf{u}(t)=\left(u_{1}(t), \cdots, u_{m}(t)\right)^{\top}$ and $c(t)$ are called investment and consumption strategies, respectively. Also, we let $Y^{\mathbf{u}, c}(t)$ be the total wealth at time $t$ under the investment and consumption strategies $(\mathbf{u}(\cdot), c(\cdot))$. Given the dynamics of the risk-free and risky assets as described in (2.3) and (2.6), respectively, the wealth 
process, $Y^{\mathbf{u}, c}(t)$ satisfies the following SDE:

$$
d Y^{\mathbf{u}, c}(t)=\left[r(t) Y^{\mathbf{u}, c}(t)+\mathbf{u}^{\top}(t) \boldsymbol{\beta}(t)-c(t)\right] d t+\mathbf{u}^{\top}(t) \boldsymbol{\sigma}(t) d \mathbf{W}(t), \quad t \in[0, T],
$$

where

$$
\boldsymbol{\beta}(t)=\boldsymbol{\alpha}(t)-r(t) \mathbf{1}_{m \times 1}=\boldsymbol{\theta}(t)-\mathbf{A X}(t)+\frac{1}{2} \mathcal{D}(\boldsymbol{\Sigma}(t)) \mathbf{1}_{m \times 1}-r(t) \mathbf{1}_{m \times 1} .
$$

Using Ito's formula again, the dynamics of the risk premium can be found as

$$
d \boldsymbol{\beta}(t)=[\boldsymbol{\Theta}(t)-\mathbf{A} \boldsymbol{\beta}(t)] d t-\mathbf{A} \boldsymbol{\sigma}(t) d \mathbf{W}(t),
$$

where

$$
\boldsymbol{\Theta}(t)=\frac{d \boldsymbol{\theta}(t)}{d t}+\left\{\frac{1}{2}\left[\frac{d \mathcal{D}(\boldsymbol{\Sigma}(t))}{d t}+\mathbf{A} \mathcal{D}(\boldsymbol{\Sigma}(t)]-\frac{d r(t)}{d t} \mathbf{I}_{m}-\mathbf{A} r(t)\right\} \mathbf{1}_{m \times 1}\right.
$$

The investor's problem is to choose an admissible investment and consumption pair $(\mathbf{u}(\cdot), c(\cdot))$ to maximize his expected utility from the intermediate consumption and the terminal wealth. Mathematically, such an objective functional is stated as:

$$
\mathbf{E}\left[\int_{0}^{T} e^{-\int_{0}^{s} \rho(l) d l} U(c(s)) d s+e^{-\int_{0}^{T} \rho(s) d s} B\left(Y^{\mathbf{u}, c}(T)\right)\right]
$$

where $\mathbf{E}$ denotes the expectation operator under the probability measure $\mathbf{P}$. In $(2.11), \rho(t)$ is the subjective discount rate, which is assumed to be a deterministic function of time $t$ and bounded on $[0, T] . U(c)$ and $B(x)$ are two utility functions measuring the utility from the intermediate consumption $c(t)$ and the terminal wealth $Y(T)$, respectively. In general, $B(x)$ and $U(c)$ can be of different forms. For the sake of analytical tractability, $U(c)$ and $B(x)$ are assumed to be of the same form in Xia (2001), Wachter (2002) and Liu (2007). In this paper, we assume that both of them are taking the exponential form as

$$
U(c)=-\frac{1}{\eta} e^{-\eta c}, \quad B(x)=-\frac{1}{\eta} e^{-\eta x}, \quad \eta>0,
$$

where $\eta$ is the risk-aversion parameter of the investor.

In fact, a similar optimization problem with an exponential utility function defined on the 
terminal wealth has been studied by Tourin and Yan (2013). However, the utility coming from the intermediate consumption is ignored there. Until very recently, Shen and Siu (2017) included the utility from consumption under a cointegration model and solved the corresponding optimization problem with a verification theorem. However, their verification theorem is invalid when the utility function is of exponential form. In this paper, we explore the optimal investment and consumption problem under the cointegration model with an exponential utility function.

Before looking for the optimal pair, we first define the admissible set of investment and consumption strategies.

Definition 1. An investment and consumption pair $(\mathbf{u}(\cdot), c(\cdot))$ is admissible, if the following conditions are satisfied.

1. The investment strategy $\mathbf{u}(\cdot)$ is an $\mathbf{R}^{m}$ valued, $\mathcal{F}_{t}$ adapted process such that

$$
\mathbf{E} \int_{0}^{T}\|\mathbf{u}(t)\|_{m}^{2} d t<\infty
$$

where $\|\cdot\|_{m}$ denotes the Euclidean norm in $\mathbf{R}^{\mathbf{m}}$.

2. The consumption strategy $c(\cdot)$ is an $\mathbf{R}$ valued, $\mathcal{F}_{t}$ adapted process such that

$$
\mathbf{E} \int_{0}^{T}|c(t)| d t<\infty
$$

3. The corresponding objective functional is finite, i.e.

$$
\mathbf{E}\left[\left|\int_{0}^{T} e^{-\int_{0}^{s} \rho(u) d u} U(c(s)) d s+e^{-\int_{0}^{T} \rho(l) d l} B\left(Y^{\mathbf{u}, c}(T)\right)\right|\right]<\infty .
$$

The class of all such admissible pairs is denote by $\mathcal{A}$.

Remark 1. The admissible set $\mathcal{A}$ is different from the one associated with a power or logarithmic utility function defined on $\mathbf{R}^{+}$(Lim et al., 2011; Shen and Siu, 2017; Zhu and Ma, 2018). Since the exponential utility function is defined on $\mathbf{R}$, the wealth process $Y^{\mathbf{u}, c}(t)$ is no longer required to be positive. Actually, removing such a constraint is reasonable. For example, an investor may consume a lot in advance to enjoy the utility from consumption, which may force the wealth 
process to be negative for short periods within $[0, T]$. Such a case lies in our admissible set $\mathcal{A}$, while it has been excluded with a power or logarithmic utility function.

In a brief summary, the original optimal investment and consumption problem has now been reformulated as an optimal stochastic control problem with the objective functional (2.11), the dynamics of the wealth $(2.7)$ and the risk-premium (2.9) to find the optimal strategies $\left(\mathbf{u}^{*}(\cdot), c^{*}(\cdot)\right)$ over the admissible set $\mathcal{A}$. An analytical closed-form solution for the problem is derived and discussed in the next section.

\section{The optimal solution and the verification theorem}

\subsection{The optimal solution}

In this section, we apply the dynamic programming method to seek the solution of the optimal stochastic control problem formulated in the previous section.

Consider a dynamic version of the cost functional defined as

$$
\mathbf{J}(t, y, \boldsymbol{\beta} ; \mathbf{u}(\cdot), c(\cdot))=\mathbf{E}_{t}\left[\int_{t}^{T} e^{-\int_{t}^{s} \rho(l) d l} U(c(s)) d s+e^{-\int_{t}^{T} \rho(l) d l} U\left(Y^{\mathbf{u}, c}(T)\right)\right],
$$

where $\mathbf{E}_{t}$ denotes the conditional expectation on $Y(t)=y, \boldsymbol{\beta}(t)=\boldsymbol{\beta}$. The value function of the optimal stochastic control problem is defined as

$$
V(t, y, \boldsymbol{\beta})=\max _{(\mathbf{u}(\cdot), c(\cdot)) \in \mathcal{A}} \mathbf{J}(t, y, \boldsymbol{\beta} ; \mathbf{u}(\cdot), c(\cdot))
$$

We assume that there exists a smooth value function $V(t, y, \boldsymbol{\beta}) \in \mathcal{C}^{1,2,2}\left([0, T] \times \mathbf{R} \times \mathbf{R}^{m}\right)$ satisfying the HJB equation:

$$
\left\{\begin{array}{l}
\max _{(\mathbf{u}, c) \in \mathbf{R}^{m} \times \mathbf{R}}\left[\mathcal{L}^{\mathbf{u}, c} V(t, y, \boldsymbol{\beta})+U(c)\right]=0, \quad(t, y, \boldsymbol{\beta}) \in[0, T] \times \mathbf{R} \times \mathbf{R}^{m} \\
V(T, y, \boldsymbol{\beta})=U(y)
\end{array}\right.
$$


where

$$
\begin{aligned}
\mathcal{L}^{\mathbf{u}, c} V(t, y, \boldsymbol{\beta}) & =\frac{\partial V}{\partial t}+\left[r(t) y+\mathbf{u}^{\top} \boldsymbol{\beta}-c\right] \frac{\partial V}{\partial y}+\frac{1}{2} \mathbf{u}^{\top} \boldsymbol{\Sigma}(t) \mathbf{u} \frac{\partial^{2} V}{\partial y^{2}}+\left(\frac{\partial V}{\partial \boldsymbol{\beta}}\right)^{\top}[\boldsymbol{\Theta}(t)-\mathbf{A} \boldsymbol{\beta}] \\
& +\frac{1}{2} \operatorname{tr}\left(\frac{\partial^{2} V}{\partial \boldsymbol{\beta}^{2}} \mathbf{A} \boldsymbol{\Sigma}(t) \mathbf{A}^{\top}\right)-\mathbf{u}^{\top} \boldsymbol{\Sigma}(t) \mathbf{A}^{\top} \frac{\partial^{2} V}{\partial y \partial \boldsymbol{\beta}}-\rho V
\end{aligned}
$$

with $\operatorname{tr}(\cdot)$ denoting the trace operator. Motivated by the method of separation of variables adopted by Merton (1969), we consider the following form as a trial solution

$$
V(t, y, \boldsymbol{\beta})=-\frac{g(t, \boldsymbol{\beta})}{\eta} e^{a(t) y}
$$

where $g(t, \boldsymbol{\beta}) \in \mathcal{C}^{1,2}\left([0, T] \times \mathbf{R}^{m}\right)$ and $a(t) \in \mathcal{C}^{1}([0, T])$. Naturally, $g(t, \beta)$ and $a(t)$ satisfy the terminal conditions $g(T, \boldsymbol{\beta})=1$ and $a(T)=-\eta$, respectively.

Lemma 1. Suppose that $g(t, \boldsymbol{\beta}) \in \mathcal{C}^{1,2}\left([0, T] \times \mathbf{R}^{m}\right)$ is positive and $a(t) \in \mathcal{C}^{1}([0, T])$. The candidates for the optimal investment and consumption strategies are given by

$$
\left\{\begin{array}{l}
\mathbf{u}^{*}(t, y, \boldsymbol{\beta})=\frac{1}{g(t, \boldsymbol{\beta}) a(t)}\left[\mathbf{A}^{\top} \frac{\partial g(t, \boldsymbol{\beta})}{\partial \boldsymbol{\beta}}-\boldsymbol{\Sigma}^{-1}(t) \boldsymbol{\beta} g(t, \boldsymbol{\beta})\right] \\
c^{*}(t, y, \boldsymbol{\beta})=-\frac{a(t) y}{\eta}-\frac{1}{\eta} \ln \left[-\frac{1}{\eta} a(t) g(t, \boldsymbol{\beta})\right]
\end{array}\right.
$$

Proof. To achieve the regular interior maximum in (3.3) with respect to $(\mathbf{u}, c)$, we set the corresponding first-order derivatives to be zero.

$$
\left\{\begin{array}{l}
\frac{\partial}{\partial \mathbf{u}}\left[\mathcal{L}^{\mathbf{u}, c} V(t, y, \boldsymbol{\beta})+U(c)\right]=\boldsymbol{\beta} \frac{\partial V(t, y, \boldsymbol{\beta})}{\partial y}+\boldsymbol{\Sigma}(t) \mathbf{u} \frac{\partial^{2} V(t, y, \boldsymbol{\beta})}{\partial y^{2}}-\boldsymbol{\Sigma}(t) \mathbf{A}^{\top} \frac{\partial^{2} V(t, y, \boldsymbol{\beta})}{\partial y \partial \boldsymbol{\beta}}=\mathbf{0}_{m \times 1} \\
\frac{\partial}{\partial c}\left[\mathcal{L}^{\mathbf{u}, c} V(t, y, \boldsymbol{\beta})+U(c)\right]=-\frac{\partial V(t, y, \boldsymbol{\beta})}{\partial y}+\frac{\partial U(c)}{\partial c}=0
\end{array}\right.
$$

Substituting the trial solution (3.5) into (3.7) results in (3.6). In addition, we also need to check the second-order conditions to any regular interior maximum. After some simple calculations, we obtain

$$
\left\{\begin{array}{l}
\frac{\partial^{2}}{\partial \mathbf{u}^{2}}\left[\mathcal{L}^{\mathbf{u}^{*}, c^{*}} V(t, y, \boldsymbol{\beta})+U(c)\right]=\boldsymbol{\Sigma}(t) \frac{\partial^{2} V(t, y, \boldsymbol{\beta})}{\partial \boldsymbol{\beta}^{2}}=-\frac{g(t, \boldsymbol{\beta})}{\eta} e^{a(t) y} a^{2}(t) \boldsymbol{\Sigma}(t), \\
\frac{\partial^{2}}{\partial c^{2}}\left[\mathcal{L}^{\mathbf{u}^{*}, c^{*}} V(t, y, \boldsymbol{\beta})+U(c)\right]=\frac{\partial^{2} U\left(c^{*}\right)}{\partial c^{2}}=-\eta e^{-\eta c^{*}}
\end{array}\right.
$$

It should be remarked that $\frac{\partial^{2}}{\partial \mathbf{u}^{2}}\left[\mathcal{L}^{\mathbf{u}, c} V(t, y, \boldsymbol{\beta})+U(c)\right]$ is negative definite for all $t \in[0, T]$ because $g(t, \boldsymbol{\beta})$ and $a^{2}(t)$ are positive and $\boldsymbol{\Sigma}(t)$ is positive definite. Moreover, $\frac{\partial^{2}}{\partial c^{2}}\left[\mathcal{L}^{\mathbf{u}, c} V(t, y, \boldsymbol{\beta})+U(c)\right]$ 
is negative definite since the utility function $U(c)$ is strictly concave. In addition, the cross-term derivative is obviously zero. After verifying the second-order conditions, we come to a conclusion that the pair $\left(\mathbf{u}^{*}, c^{*}\right)$ given in $(3.6)$ is indeed the global maximum point. This completes the proof.

Remark 2. The candidate optimal pair $\left(\mathbf{u}^{*}, c^{*}\right)$ obtained in Lemma 1 is the global maximum point but may not be located in the admissible set $\mathcal{A}$ defined by Definition 1 . Its admissibility is yet to be verified in Lemma 4 later, after their analytical expressions are derived in Theorem 1.

In Lemma 2, we provide a characterization for the unknown functions $a(t)$ and $g(t, \boldsymbol{\beta})$.

Lemma 2. Suppose that $a(t) \in \mathcal{C}^{1}([0, T])$ and $g(t, \boldsymbol{\beta}) \in \mathcal{C}^{1,2}\left([0, T] \times \mathbf{R}^{m}\right)$. Then, $a(t)$ satisfies a Riccati differential equation as

$$
\frac{d a(t)}{d t}+r(t) a(t)+\frac{a^{2}(t)}{\eta}=0
$$

and $g(t, \boldsymbol{\beta})$ satisfies

$$
\begin{aligned}
\frac{\partial g(t, \boldsymbol{\beta})}{\partial t} & =\left[\rho(t)+\frac{1}{2} \boldsymbol{\beta}^{\top} \boldsymbol{\Sigma}^{-1} \boldsymbol{\beta}+\frac{a(t)}{\eta}\right] g(t, \boldsymbol{\beta})-\frac{a(t) g(t, \boldsymbol{\beta})}{\eta} \ln \left[-\frac{a(t) g(t, \boldsymbol{\beta})}{\eta}\right]-\left(\frac{\partial g(t, \boldsymbol{\beta})}{\partial \boldsymbol{\beta}}\right)^{\top} \boldsymbol{\Theta}(t) \\
& +\frac{1}{2 g(t, \boldsymbol{\beta})}\left(\frac{\partial g(t, \boldsymbol{\beta})}{\partial \boldsymbol{\beta}}\right)^{\top} \mathbf{A} \boldsymbol{\Sigma}(t) \mathbf{A}^{\top} \frac{\partial g(t, \boldsymbol{\beta})}{\partial \boldsymbol{\beta}}-\frac{1}{2} \operatorname{tr}\left(\frac{\partial^{2} g(t, \boldsymbol{\beta})}{\partial \boldsymbol{\beta}^{2}} \mathbf{A} \boldsymbol{\Sigma}(t) \mathbf{A}^{\top}\right),
\end{aligned}
$$

with the terminal conditions $a(T)=-\eta$ and $g(T, \boldsymbol{\beta})=1$, respectively.

Proof. Substituting (3.6) into the HJB equation (3.3) leads to

$$
\begin{aligned}
0= & g(t)\left[\frac{d a(t)}{d t}+r(t) a(t)+\frac{a^{2}(t)}{\eta}\right] y-\left[\rho(t)+\frac{1}{2} \boldsymbol{\beta}^{\top} \boldsymbol{\Sigma}^{-1}(t) \boldsymbol{\beta}+\frac{a(t)}{\eta}\right] g(t, \boldsymbol{\beta}) \\
& +\frac{a(t) g(t, \boldsymbol{\beta})}{\eta} \ln \left[-\frac{a(t) g(t, \boldsymbol{\beta})}{\eta}\right]+\left(\frac{\partial g(t, \boldsymbol{\beta})}{\partial \boldsymbol{\beta}}\right)^{\top} \boldsymbol{\Theta}(t)+\frac{\partial g(t, \boldsymbol{\beta})}{\partial t}+ \\
& -\frac{1}{2 g(t, \boldsymbol{\beta})}\left(\frac{\partial g(t, \boldsymbol{\beta})}{\partial \boldsymbol{\beta}}\right)^{\top} \mathbf{A} \Sigma(t) \mathbf{A}^{\top} \frac{\partial g(t, \boldsymbol{\beta})}{\partial \boldsymbol{\beta}} \frac{1}{2} \operatorname{tr}\left(\frac{\partial^{2} g(t, \boldsymbol{\beta})}{\partial \boldsymbol{\beta}^{2}} \mathbf{A} \Sigma(t) \mathbf{A}^{\top}\right) .
\end{aligned}
$$

By matching the coefficient of $y$ and the remaining term, the ordinary differential equation (ODE) in (3.9) and the partial differential equation (PDE) in (3.10) are derived directly.

In the following lemma, we derive the analytical formula for functions $a(t)$ and $g(t, \boldsymbol{\beta})$. 
Lemma 3. The solution to the Riccati differential equation (3.9) is given by

$$
a(t)=-\eta h(t)
$$

where $h(t)=\frac{1}{e^{-\int_{t}^{T} r(s) d s}\left(1+\int_{t}^{T} e^{\int_{s}^{T} r(u) d u} d s\right)}$. The second-order PDE (3.10) admits an analytical solution

$$
g(t, \boldsymbol{\beta})=e^{\left\{K_{1}(t)+\mathbf{K}_{2}^{\top}(t) \boldsymbol{\beta}+\frac{1}{2} \boldsymbol{\beta}^{\top} \mathbf{K}_{3}(t) \boldsymbol{\beta}\right\}},
$$

where

$$
\left\{\begin{array}{l}
K_{1}(t)=\int_{t}^{T} e^{-\int_{t}^{s} h(u) d u}\left[\mathbf{K}_{2}^{\top}(s) \boldsymbol{\Theta}(s)+\frac{1}{2} \operatorname{tr}\left(\mathbf{K}_{3}(s) \mathbf{A} \Sigma(s) \mathbf{A}^{\top}\right)-\rho(s)+h(s)(1-\ln h(s))\right] d s, \\
\mathbf{K}_{2}(t)=\int_{t}^{T} e^{-\int_{t}^{s} h(u) d u} \mathbf{K}_{3}(s) \boldsymbol{\Theta}(s) d s \\
\mathbf{K}_{3}(t)=-\int_{t}^{T} e^{-\int_{t}^{s} h(u) d u} \boldsymbol{\Sigma}^{-1}(s) d s .
\end{array}\right.
$$

Proof. By introducing a transformation $q(t)=\frac{1}{a(t)}$, the Riccati differential equation (3.9) becomes a linear ODE, i.e.

$$
\frac{d q(t)}{d t}=r(t) q(t)+\frac{1}{\eta}
$$

with $q(T)=-\frac{1}{\eta}$. The solution can be easily obtained as

$$
q(t)=-\frac{1}{\eta} e^{-\int_{t}^{T} r(s) d s}\left(1+\int_{t}^{T} e^{\int_{s}^{T} r(u) d u} d s\right)
$$

As a result, we have $a(t)=-\eta h(t)$ where $h(t)=\frac{1}{e^{-\int_{t}^{T} r(s) d s}\left(1+\int_{t}^{T} e^{\int_{s}^{T} r(u) d u} d s\right)}$. Then, we can solve the PDE (3.10) with a trial solution in the form (3.12). Substituting it into (3.10) leads to

$$
\begin{array}{r}
0=\frac{d K_{1}(t)}{d t}-h(t) \mathbf{K}_{1}(t)+\mathbf{K}_{2}^{\top}(t) \boldsymbol{\Theta}(t)+\frac{1}{2} \operatorname{tr}\left(\mathbf{K}_{3}(t) \mathbf{A} \boldsymbol{\Sigma}(t) \mathbf{A}^{\top}\right)-[\rho(t)-h(t)+h(t) \ln h(t)] \\
+\boldsymbol{\beta}^{T}\left[\frac{d \mathbf{K}_{3}(t)}{d t}-h(t) \mathbf{K}_{3}(t)-\boldsymbol{\Sigma}^{-1}(t)\right] \boldsymbol{\beta}+\left[\frac{d \mathbf{K}_{2}(t)}{d t}-h(t) \mathbf{K}_{2}(t)+\mathbf{K}_{3}(t) \boldsymbol{\Theta}(t)\right]^{\top} \boldsymbol{\beta} .
\end{array}
$$


Again, matching the coefficients for $\boldsymbol{\beta}^{\top}(\cdot) \boldsymbol{\beta}, \boldsymbol{\beta}^{\top}(\cdot)$ and the constant yields an ODE system

$$
\left\{\begin{array}{l}
\frac{d K_{1}(t)}{d t}=h(t) \mathbf{K}_{1}(t)-\mathbf{K}_{2}^{\top}(t) \boldsymbol{\Theta}(t)-\frac{1}{2} \operatorname{tr}\left(\mathbf{K}_{3}(t) \mathbf{A} \boldsymbol{\Sigma}(t) \mathbf{A}^{\top}\right)+\rho(t)-h(t)+h(t) \ln h(t), \\
\frac{d \mathbf{K}_{2}(t)}{d t}=h(t) \mathbf{K}_{2}(t)-\mathbf{K}_{3}(t) \boldsymbol{\Theta}(t) \\
\frac{d \mathbf{K}_{3}(t)}{d t}=h(t) \mathbf{K}_{3}(t)+\boldsymbol{\Sigma}^{-1}(t)
\end{array}\right.
$$

with the terminal conditions $K_{1}(T)=0, \mathbf{K}_{2}(T)=\mathbf{0}_{m \times 1}, \mathbf{K}_{3}(T)=\mathbf{0}_{m \times m}$. Sine the PDE system (3.16) is linear, the corresponding solutions are easily produced as shown in (3.13) by applying the method of variation of parameters.

Combining Lemmas 1-3, the main results of this paper can be summarized in the following theorem.

Theorem 1. When asset prices follow the cointegration model (2.5), the candidate optimal investment and consumption strategies for an investor with exponential utility are given by

$$
\left\{\begin{array}{l}
\mathbf{u}^{*}(t, \boldsymbol{\beta})=\frac{1}{\eta h(t)} \mathbf{\Sigma}(t)^{-1} \boldsymbol{\beta}-\frac{1}{\eta h(t)} \mathbf{A}^{\top}\left[\mathbf{K}_{3}(t) \boldsymbol{\beta}+\mathbf{K}_{2}(t)\right] \\
c^{*}(t, y, \boldsymbol{\beta})=h(t) y-L(t, \boldsymbol{\beta})
\end{array}\right.
$$

where $L(t, \boldsymbol{\beta})=\frac{1}{\eta}\left[K_{1}(t)+\mathbf{K}_{2}^{\top}(t) \boldsymbol{\beta}+\frac{1}{2} \boldsymbol{\beta}^{\top} \mathbf{K}_{3}(t) \boldsymbol{\beta}\right]$. The solution of the HJB equation (3.3) admits an analytical expression as

$$
V(t, y, \boldsymbol{\beta})=-\frac{1}{\eta} e^{\left\{-\eta h(t) y+K_{1}(t)+\mathbf{K}_{2}^{\top}(t) \boldsymbol{\beta}+\frac{1}{2} \boldsymbol{\beta}^{\top} \mathbf{K}_{3}(t) \boldsymbol{\beta}\right\}} .
$$

Remark 3. It is easy to verify that functions $a(t)$ and $g(t, \boldsymbol{\beta})$ derived in Lemma 3 satisfy all the assumptions stated in Lemmas 1 and 2. The value function obtained in Theorem 1 also satisfies the smooth assumption, i.e. $V(t, y, \boldsymbol{\beta}) \in \mathcal{C}^{1,2,2}\left([0, T] \times \mathbf{R} \times \mathbf{R}^{m}\right)$. From (3.13), we observe that $\mathbf{K}_{3}(t)$ is negative semi-definite for all $t \in[0, T]$ as $\boldsymbol{\Sigma}^{-1}(t)$ is positive definite. In addition, $h(t)$, $K_{1}(t), \mathbf{K}_{2}(t)$ and $\mathbf{K}_{3}(t)$ are all bounded since $r(t), \boldsymbol{\theta}(t), \boldsymbol{\sigma}(t), \boldsymbol{\Sigma}(t)$ and $\boldsymbol{\Sigma}^{-1}(t)$ are assumed to be deterministic functions and bounded on $[0, T]$.

As the main contribution of this paper, Theorem 1 provides closed-form analytical formula for the optimal strategies and the value function so that we can easily carry out discussions later on how cointegration boosts the optimal expected utility for the investor. 


\subsection{The verification theorem}

Before carrying out discussions, we should establish a verification theorem first. As pointed out by Korn and Kraft (2004), even though the analytical solution of the HJB equation has been derived, it is still necessary to check all the sufficient conditions, especially in a stochastic environment. The classical verification theorem proposed by Fleming and Soner (2006) does not work in our case because the value function (3.18) does not satisfy the polynomial growth condition. Therefore, we need to establish a new and proper verification theorem to demonstrate that the solution of the HJB equation (3.18) is indeed the solution of the original optimization problem. First of all, let us verify the admissibility of the candidate optimal pair $\left(\mathbf{u}^{*}(\cdot), c^{*}(\cdot)\right)$ in the following lemma.

Lemma 4. If the process $\boldsymbol{\beta}(t)$ satisfies the following integrability condition:

$$
\mathbf{E} e^{C \int_{0}^{T}\|\boldsymbol{\beta}(t)\|_{m}^{2} d t}<\infty
$$

where $C$ is a positive constant ${ }^{1}$, then the candidate optimal pair $\left(\mathbf{u}^{*}(\cdot), c^{*}(\cdot)\right)$ given by $(3.17)$ is admissible. In addition, for any admissible pair $(\mathbf{u}(\cdot), c(\cdot))$, we can conclude that the following family

$$
\left\{V\left(\tau, Y^{\mathbf{u}, c}(\tau), \boldsymbol{\beta}(\tau)\right)\right\}_{\tau \in \mathcal{K}}
$$

is uniformly integrable, where $\mathcal{K}$ denotes the set of all stopping times within the interval $0 \leq \tau \leq$ $T$.

Proof. In order to check the admissibility of the optimal pair $\left(\mathbf{u}^{*}(\cdot), c^{*}(\cdot)\right)$ given by $(3.17)$, we need to verify the conditions (2.13), (2.14) and (2.15) in Definition 1, respectively.

First of all, we verify the integrable conditions (2.13) and (2.14). From (3.17), it suffices to prove the integrability of the processes $\boldsymbol{\beta}(t)$ and $Y^{\mathbf{u}^{*}, c^{*}}(t)$. The proof of the integrability of $\boldsymbol{\beta}(t)$ starts with the expression

$$
\boldsymbol{\beta}(t)=\underbrace{e^{-\mathbf{A} t} \boldsymbol{\beta}_{0}+\int_{0}^{t} e^{\mathbf{A}(s-t)} \boldsymbol{\Theta}(s) d s}_{\boldsymbol{\mu}(t)}-\underbrace{\int_{0}^{t} e^{\mathbf{A}(s-t)} \mathbf{A} \boldsymbol{\sigma}(s) d \mathbf{W}(s)}_{\mathbf{H}(t)},
$$

\footnotetext{
${ }^{1}$ Such a constant will be given later.
} 
where $\mathbf{H}(t) \sim \mathcal{N}\left(\mathbf{0}_{m \times 1}, \boldsymbol{\Sigma}_{\mathbf{H}}(t)\right)$ and $\left.\boldsymbol{\Sigma}_{\mathbf{H}}(t)\right)=\int_{0}^{t} e^{\mathbf{A}(s-t)} \mathbf{A} \boldsymbol{\sigma}(s) \boldsymbol{\sigma}^{\top}(s) \mathbf{A}^{\top} e^{\mathbf{A}^{\top}(s-t)} d s$. Obviously, $\boldsymbol{\beta}(t)$ has a finite second moment because $\boldsymbol{\Theta}(t)$ and $\boldsymbol{\sigma}(t)$ are bounded on $[0, T]$. Furthermore, according to Yong and Zhou (1999), we have, for any $p \geq 1$, an estimate

$$
\mathbf{E} \max _{0 \leq s \leq T}\|\boldsymbol{\beta}(s)\|_{m}^{p} \leq C_{1}\left(1+\|\boldsymbol{\beta}(0)\|_{m}^{p}\right)
$$

where $C_{1}$ is a positive constant. As a result, the processes $\boldsymbol{\beta}(t)$ is integrable, which indicates that the optimal investment strategy $\mathbf{u}^{*}(t)$ satisfies the integrable condition (2.13).

On the other hand, substituting the optimal pair $\left(\mathbf{u}^{*}(\cdot), c^{*}(\cdot)\right)$ back into the dynamics of wealth process $(2.7)$ results in

$$
d Y^{\mathbf{u}^{*}, c^{*}}(t)=\left[A_{1}(t) Y^{\mathbf{u}^{*}, c^{*}}(t)+B_{1}(t)\right] d t+\mathbf{D}_{1}(t) d \mathbf{W}(t)
$$

where

$$
\left\{\begin{aligned}
A_{1}(t) & =r(t)-h(t) \\
B_{1}(t) & =\frac{\boldsymbol{\beta}^{T}(t)\left[\boldsymbol{\Sigma}^{-1}(t)-\mathbf{A}^{\top} \mathbf{K}_{3}(t)+h(t) \mathbf{K}_{3}(t)\right] \boldsymbol{\beta}(t)+\left[h(t) \mathbf{K}_{2}^{\top}(t)-\mathbf{K}_{2}^{\top}(t) \mathbf{A}\right] \boldsymbol{\beta}(t)+h(t) \ln h(t)}{\eta h(t)} \\
\mathbf{D}_{1}(t) & =\frac{\boldsymbol{\beta}^{\top}(t)\left[\boldsymbol{\Sigma}^{-1}(t)-\mathbf{K}_{3}(t) \mathbf{A}\right] \boldsymbol{\sigma}(t)-\mathbf{K}_{2}^{\top}(t) \mathbf{A} \boldsymbol{\sigma}(t)}{\eta h(t)}
\end{aligned}\right.
$$

Based on the estimate (3.22) and the boundedness of functions $r(t), h(t), \boldsymbol{\Sigma}^{-1}(t), K_{1}(t), \mathbf{K}_{2}(t)$, and $\mathbf{K}_{3}(t)$, we know that $A_{1}(t)$ is bounded on $[0, T]$ and

$$
B_{1}(t) \in L_{\mathcal{F}}^{2}(0, T ; \mathbf{R}), \mathbf{D}_{1}(t) \in L_{\mathcal{F}}^{2}\left(0, T ; \mathbf{R}^{m}\right),
$$

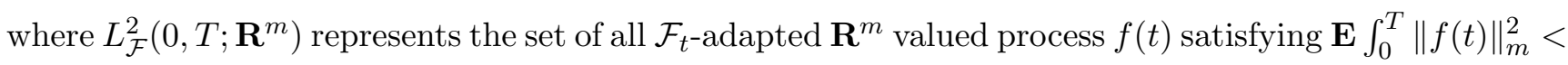
$\infty$. Again, according to Yong and Zhou (1999), the SDE (3.23) admits a unique strong solution

$$
Y^{\mathbf{u}^{*}, c^{*}}(t)=e^{l(t)}\left[Y(0)+\int_{0}^{t} e^{-l(s)} B_{1}(s) d s+\int_{0}^{t} e^{-l(s)} \mathbf{D}_{1}(s) d \mathbf{W}(s)\right]
$$

where $l(t)=\int_{0}^{t} A_{1}(s) d s$ is bounded on $[0, T]$. Similarly, it has an estimated upper bound

$$
\mathbf{E} \max _{0 \leq s \leq T}\left|Y^{\mathbf{u}^{*}, c^{*}}(s)\right|^{p} \leq C_{2}\left(1+|Y(0)|^{p}\right),
$$


where $C_{2}$ is a positive constant. Consequently, the integrability of process $Y^{\mathbf{u}^{*}, c^{*}}(t)$ is also verified, which leads to the conclusion that the optimal consumption strategy $c^{*}(t)$ satisfies the integrable condition (2.14).

Now let us verify the condition (2.15). Since $\mathbf{K}_{3}(t)$ is negative semi-definite as shown in Remark 3 , the quadratic term $L(t, \boldsymbol{\beta}(t))$ is bounded above as

$$
\mathbf{E} e^{-\eta c^{*}(t)}=\mathbf{E} e^{-\eta h(t) Y^{\mathbf{u}^{*}, c^{*}}(t)+\eta L(t, \boldsymbol{\beta}(t))} \leq C_{3} \mathbf{E} e^{-\eta h(t) Y^{\mathbf{u}^{*}, c^{*}(t)},}
$$

where $C_{3}$ is a positive constant. Furthermore, we have

$$
\begin{aligned}
& \mathbf{E}\left[\mid \int_{0}^{T} e^{-\int_{0}^{s} \rho(l) d l} U\left(c^{*}(s)\right) d s+e^{-\int_{0}^{T} \rho(l) d l} U\left(Y^{\mathbf{u}^{*}, c^{*}}(T) \mid\right]\right. \\
& =\frac{1}{\eta}\left[\int_{0}^{T} e^{-\int_{0}^{s} \rho(l) d l} \mathbf{E} e^{-\eta c^{*}} d s+e^{-\int_{0}^{T} \rho(l) d l} \mathbf{E} e^{-\eta Y^{\mathbf{u}^{*}, c^{*}}(T)}\right] \\
& \leq C_{4}\left[\int_{0}^{T} \mathbf{E} e^{-\eta c^{*}} d s+\mathbf{E} e^{-\eta Y^{\mathbf{u}^{*}, c^{*}}(T)}\right] \\
& \leq C_{5}\left[\int_{0}^{T} \mathbf{E} e^{-\eta h(s) Y^{\mathbf{u}^{*}, c^{*}(s)}} d s+\mathbf{E} e^{-\eta Y^{\mathbf{u}^{*}, c^{*}}(T)}\right],
\end{aligned}
$$

where $C_{4}, C_{5}$ are two different positive constants. The objective functional (3.29) is finite, if we can prove that the family $\left\{e^{-\eta h(\tau) Y^{\mathbf{u}^{*}, c^{*}}(\tau)}\right\}_{\tau \in \mathcal{K}}$ is uniformly integrable, i.e.

$$
\mathbf{E} e^{-\eta h(\tau) Y^{\mathbf{u}^{*}, c^{*}(\tau)}}<\infty, \quad \tau \in \mathcal{K}
$$

In order to prove the uniform integrability of the family above, we first define a new stochastic process $M(t) \triangleq-2 \eta h(t) \int_{0}^{t} e^{l(t)-l(s)} \mathbf{D}_{1}(s) d \mathbf{W}(s)$ and then construct an exponential martingale as

$$
Z(t) \triangleq e^{M(t)-\frac{1}{2}\langle M\rangle_{t}}
$$

where $\langle M\rangle_{t}$ denotes the predictable quadratic variation process of the process $M(t)$. So far, $Z(t)$ is only a local martingale. For the newly defined process $M(t)$, we have

$$
\mathbf{E}\left[e^{\frac{1}{2}\langle M\rangle_{T}}\right]=\mathbf{E}\left[e^{2 \eta^{2} \int_{0}^{T} e^{2 l(T)-2 l(t)}\left\|\mathbf{D}_{1}(t)\right\|_{m}^{2} d t}\right] \leq C_{6} \mathbf{E} e^{C_{7} \int_{0}^{T}\|\boldsymbol{\beta}(t)\|_{m}^{2} d t}
$$


where $C_{6}$ and $C_{7}$ are two different positive constants. If the condition (3.19) holds and $C \geq C_{7}$ is satisfied, then (3.32) is finite, i.e. the Novikov condition is satisfied. Consequently, $Z(t)$ becomes a martingale and we have $\mathbf{E} Z(t)=Z(0)$ for $t \in[0, T]$, which results in

$$
\mathbf{E} e^{M(t)}=\mathbf{E} e^{-2 \eta h(t) e^{l(t)} \int_{0}^{t} e^{-l(s)} \mathbf{D}_{1}(s) d \mathbf{W}(s)}=\mathbf{E} e^{2 \eta^{2} h^{2}(t) e^{2 l(t)} \int_{0}^{t} e^{-2 l(s)}\left\|\mathbf{D}_{1}(s)\right\|_{m}^{2} d s}=\mathbf{E} e^{\frac{1}{2}\langle M\rangle_{t}}
$$

Now, we are ready to prove the uniform integrability of the family $\left\{e^{-\eta h(\tau) Y^{\mathbf{u}^{*}, c^{*}}(\tau)}\right\}_{\tau \in \mathcal{K}}$.

$$
\begin{aligned}
& \mathbf{E} e^{-\eta h(\tau) Y^{\mathbf{u}^{*}, c^{*}}(\tau)} \\
& \leq C_{8} \mathbf{E} e^{-\eta h(\tau)\left[\int_{0}^{\tau} e^{l(\tau)-l(s)} B_{1}(s) d s+\int_{0}^{\tau} e^{l(\tau)-l(s)} \mathbf{D}_{1}(s) d \mathbf{W}(s)\right]} \\
& \leq C_{8}\left[\mathbf{E} e^{-2 \eta h(\tau) \int_{0}^{\tau} e^{l(\tau)-l(s)} B_{1}(s) d s}\right]^{\frac{1}{2}}\left[\mathbf{E} e^{-2 \eta h(\tau) \int_{0}^{\tau} e^{l(\tau)-l(s)} \mathbf{D}_{1}(s) d \mathbf{W}(s)}\right]^{\frac{1}{2}} \quad \text { (Cauchy-Schwarz inequality) } \\
& =C_{8}\left[\mathbf{E} e^{-2 \eta h(\tau) \int_{0}^{\tau} e^{l(\tau)-l(s)} B_{1}(s) d s}\right]^{\frac{1}{2}}\left[\mathbf{E} e^{2 \eta^{2} h^{2}(\tau) \int_{0}^{\tau} e^{2 l(\tau)-2 l(s)}\left\|\mathbf{D}_{1}(s)\right\|_{m}^{2} d s}\right]^{\frac{1}{2}} \quad \text { (Equation (3.33)) } \\
& \leq C_{8}\left[\mathbf{E} e^{C_{9} \int_{0}^{\tau}\left|B_{1}(s)\right| d s}\right]^{\frac{1}{2}}\left[\mathbf{E} e^{C_{10} \int_{0}^{\tau}\left\|\mathbf{D}_{1}(s)\right\|_{m}^{2} d s}\right]^{\frac{1}{2}} \\
& \left.\leq C_{11}\left[\mathbf{E} e^{C_{12} \int_{0}^{\tau}\|\boldsymbol{\beta}(s)\|_{m}^{2} d s}\right]^{\frac{1}{2}}\left[\mathbf{E} e^{C_{13} \int_{0}^{\tau}\|\boldsymbol{\beta}(s)\|_{m}^{2} d s}\right]^{\frac{1}{2}} \quad \text { Equation }(3.24)\right) \\
& \leq C_{11} \mathbf{E} e^{C_{14} \int_{0}^{T}\|\boldsymbol{\beta}(s)\|_{m}^{2} d s},
\end{aligned}
$$

where $C_{8}, \cdots, C_{14}$ are different positive constants. If the condition (3.19) holds and $C \geq C_{14}$ is satisfied, then (3.34) is finite, which implies the uniform integrability of the family $\left\{e^{-\eta h(\tau) Y^{\mathbf{u}^{*}, c^{*}}(\tau)}\right\}_{\tau \in \mathcal{K}}$. As a direct result of the uniform integrability shown in (3.30), the objective functional (3.29) is finite, which verifies the condition (2.15).

After verifying the integrable conditions (2.13) and (2.14) and the well-defined condition (2.15), we conclude that the optimal pair $\left(\mathbf{u}^{*}(\cdot), c^{*}(\cdot)\right)$ given by $(3.17)$ is admissible.

In addition, we prove that the family $\left\{V\left(\tau, Y^{\mathbf{u}, c(\tau)}, \boldsymbol{\beta}(\tau)\right)\right\}_{\tau \in \mathcal{K}}$ is uniformly integrable. For any admissible pair $(\mathbf{u}(\cdot), c(\cdot))$, the corresponding wealth process can be derived as

$$
Y^{\mathbf{u}, c}(t)=e^{\int_{0}^{t} r(s) d s}\left[Y(0)+\int_{0}^{t} e^{-\int_{0}^{s} r(u) d u} B_{2}(s) d s+\int_{0}^{t} e^{-\int_{0}^{s} r(u) d u} \mathbf{D}_{2}(s) d \mathbf{W}(s)\right],
$$


where $B_{2}(s)=\mathbf{u}(s)^{\top} \boldsymbol{\beta}(s)-c(s)$ and $\mathbf{D}_{2}(s)=\mathbf{u}^{\top}(s) \boldsymbol{\sigma}(s)$. Similar to the proof in (3.34), we have

$$
\mathbf{E}\left[\left|V\left(\tau, Y^{\mathbf{u}, c}(\tau), \boldsymbol{\beta}(\tau)\right)\right|\right]=\frac{1}{\eta} \mathbf{E}\left[e^{-\eta h(\tau) Y^{\mathbf{u}, c}(\tau)+L(t, \boldsymbol{\beta}(\tau))}\right] \leq C_{15} e^{C_{16} \int_{0}^{T}\|\boldsymbol{\beta}(s)\|_{m}^{2} d s}
$$

where $C_{15}, C_{16}$ are two positive constants. If the condition (3.19) holds and $C \geq C_{16}$ is satisfied, then (3.36) is finite, which implies the uniform integrability of the family $\left\{V\left(\tau, Y^{\mathbf{u}, c(\tau)}, \boldsymbol{\beta}(\tau)\right)\right\}_{\tau \in \mathcal{K}}$.

Finally, from the proof above, a sufficiently large constant $C$ still needs to be specific. Now, it can be easily achieved by choosing $C=\max \left\{C_{7}, C_{14}, C_{16}\right\}$ so that the proposed condition (3.19) can guarantee that (3.32), (3.34) and (3.36) are all finite.

Remark 4. The integrability condition (3.19) is equivalent to the exponential integrability of an Ornstein-Uhlenbeck process $\boldsymbol{\beta}(t)$ and its time integral. In general, an Ornstein-Uhlenbeck process only satisfies the polynomial growth condition as shown in (3.22). Condition (3.19) does not always hold and some restrictions should be imposed to guarantee that the process $\boldsymbol{\beta}(t)$ satisfies the integrable condition (3.19).

On the other hand, (3.19) is a growth condition imposed on a stochastic process, which is difficult to verify. A condition imposed on deterministic functions may be much easier to check. For example, Benth and Karlsen (2005) imposed a simple constraint on parameters to ensure the exponential integrability of one-dimensional Ornstein-Uhlenbeck process. In the following lemma, we provide a simple and sufficient condition to ensure that the exponential integrability of the multivariate Ornstein-Uhlenbeck process $\boldsymbol{\beta}(t)$ is satisfied.

Lemma 5. (A sufficient condition) If $C$ is a positive constant satisfying

$$
4 C T \max _{0 \leq s \leq T}\left[R\left(\boldsymbol{\Sigma}_{\mathbf{H}}^{\frac{1}{2}}(s)\right)\right]^{2}<1
$$

where $R(\mathbf{O})$ denotes the spectral radius of matrix $\mathbf{O}, \mathbf{O}^{\frac{1}{2}}$ is the square root of a positive semidefinite matrix $\mathbf{O}$ and $\boldsymbol{\Sigma}_{\mathbf{H}}(t)=\int_{0}^{t} e^{\mathbf{A}(s-t)} \mathbf{A} \boldsymbol{\sigma}(s) \boldsymbol{\sigma}^{\top}(s) \mathbf{A}^{\top} e^{\mathbf{A}^{\top}(s-t)} d s$, then the integrability condition (3.19) holds. 
Proof. According to Equation (3.21), $\boldsymbol{\beta}(t)$ can be further expressed as

$$
\boldsymbol{\beta}(t)=\boldsymbol{\mu}(t)-\boldsymbol{\Sigma}_{\mathbf{H}}^{\frac{1}{2}}(t) \mathbf{Q},
$$

where $\mathbf{Q} \sim \mathcal{N}\left(\mathbf{0}_{m \times 1}, \mathbf{I}_{m}\right)$. Hence, $\mathbf{E} e^{C \int_{0}^{T}\|\boldsymbol{\beta}(t)\|_{m}^{2} d t} \leq C_{17} \mathbf{E} e^{2 C \int_{0}^{T}\left\|\boldsymbol{\Sigma}_{\mathbf{H}}^{\frac{1}{2}}(t) \mathbf{Q}\right\|_{m}^{2} d t}$, where $C_{17}$ is another positive constant. On the other hand, according to the Holder inequality and the definition of spectral radius of a matrix, we have

$$
\begin{aligned}
\mathbf{E} e^{2 C \int_{0}^{T}\left\|\boldsymbol{\Sigma}_{\mathbf{H}}^{\frac{1}{2}}(t) \mathbf{Q}\right\|_{m}^{2} d t} & =\sum_{n=0}^{\infty} \frac{(2 C)^{n}}{n !} \mathbf{E}\left(\int_{0}^{T}\left\|\boldsymbol{\Sigma}_{\mathbf{H}}^{\frac{1}{2}}(t) \mathbf{Q}\right\|_{m}^{2} d t\right)^{n} \\
& \leq \sum_{n=0}^{\infty} \frac{(2 C)^{n}}{n !} \mathbf{E}\left[\left(\int_{0}^{T} 1^{\frac{n}{n-1}} d t\right)^{\frac{n-1}{n}}\left(\int_{0}^{T}\left\|\boldsymbol{\Sigma}_{\mathbf{H}}^{\frac{1}{2}}(t) \mathbf{Q}\right\|_{m}^{2 n} d t\right)^{\frac{1}{n}}\right]^{n} \quad \text { (Holder inequality) } \\
& =\frac{1}{T} \sum_{n=0}^{\infty} \frac{(2 C T)^{n}}{n !} \mathbf{E} \int_{0}^{T}\left\|\boldsymbol{\Sigma}_{\mathbf{H}}^{\frac{1}{2}}(t) \mathbf{Q}\right\|_{m}^{2 n} d t \\
& \leq \frac{1}{T} \sum_{n=0}^{\infty} \frac{(2 C T)^{n}}{n !} \mathbf{E} \int_{0}^{T} R\left(\boldsymbol{\Sigma}_{\mathbf{H}}^{\frac{1}{2}}(t)\right)^{2 n}\|\mathbf{Q}\|_{m}^{2 n} d t \quad \text { (Definition of spectral radius) } \\
& \left.\leq \sum_{n=0}^{\infty} \frac{(2 C T)^{n}}{n !}\left[\max _{0 \leq s \leq T} R\left(\boldsymbol{\Sigma}_{\mathbf{H}}^{\frac{1}{2}}(s)\right)\right]^{2 n} \mathbf{E}\|\mathbf{Q}\|_{m}^{2 n} \quad \text { (The normality of } \mathbf{Q}\right) \\
& =\pi^{1-\frac{m}{2}} \Upsilon \sum_{n=0}^{\infty} \frac{\left.4 C T\left[\max _{0 \leq s \leq T} R\left(\boldsymbol{\Sigma}_{\mathbf{H}}^{\frac{1}{2}}(s)\right)\right]^{2}\right\}^{n}}{n !} \Gamma\left(n+\frac{m}{2}\right)
\end{aligned}
$$

where both the constant $\Upsilon$ and the function $\Gamma(n)$ are defined in Appendix A.

According to the Cauchy ratio test, when $C$ is a positive constant such that the condition (3.37) is satisfied, the series (3.39) converges, which completes the proof.

Remark 5. The sufficient condition (3.37) proposed in Lemma 5 guarantees the exponential integrability of a multivariate Ornstein-Uhlenbeck process. Of course, it can degenerate to onedimensional case by setting $m=1, A=\alpha>0$ and $\sigma(t) \equiv \sigma$. In the degenerated case, we have $\boldsymbol{\Sigma}_{\mathbf{H}}(t)=\frac{\sigma^{2}\left(1-e^{-2 \alpha t}\right)}{2 \alpha}$ and the sufficient condition (3.37) thus becomes $C<\frac{2 \alpha}{T \sigma^{2}\left(1-e^{-2 \alpha T}\right)}$, which is consistent with the condition proposed by Benth and Karlsen (2005) for the one dimensional case. Finally, compared with the integrability condition (3.19), it is much easier to check such a simple sufficient condition (3.37). 
The admissibility of the candidate optimal pair $\left(\mathbf{u}^{*}(\cdot), c^{*}(\cdot)\right)$ and the uniform integrability of the family $\left\{V\left(\tau, Y^{\mathbf{u}, c}(\tau), \boldsymbol{\beta}(\tau)\right)\right\}_{\tau \in \mathcal{K}}$ have been well demonstrated in Lemma 4 under an integrability condition (3.19). A simple and sufficient condition (3.37) is also proposed in Lemma 5, which is another contribution of this paper, to ensure such exponential integrability. Consequently, we can establish a verification theorem below and provide an important link between the solution of the HJB equation and the solution of the original optimal control problem.

Theorem 2. (Verification theorem) Suppose the assumption in Lemma 5 is enforced, then the solution to the HJB equation (3.3) is indeed the solution to the original optimization problem, i.e.

$$
V(t, y, \boldsymbol{\beta})=\max _{(u(\cdot), c(\cdot)) \in \mathcal{A}} \mathbf{J}(t, y, \boldsymbol{\beta} ; \mathbf{u}(\cdot), c(\cdot))=\mathbf{J}\left(t, y, \boldsymbol{\beta} ; \mathbf{u}^{*}(\cdot), c^{*}(\cdot)\right),
$$

and the candidates $\left(\mathbf{u}^{*}(\cdot), c^{*}(\cdot)\right)$ given in $(3.17)$ are the corresponding optimal strategies.

Proof. The outline of the proof is similar to the standard results in Øksendal (2003). For any admissible pair $(\mathbf{u}(\cdot), c(\cdot))$, we define a localized sequence of stopping times as follows:

$$
\tau^{N} \triangleq T \wedge \inf \left\{t>0|\quad| Y^{\mathbf{u}, c}(t) \mid \geq N \quad \text { or } \quad\|\boldsymbol{\beta}(t)\|_{m} \geq N\right\}, \quad N=1,2, \cdots .
$$

According to Ito's formula, we have

$$
\begin{aligned}
& e^{-\int_{t}^{\tau^{N}} \rho(l) d l} V\left(\tau^{N}, Y^{\mathbf{u}, c}\left(\tau^{N}\right), \boldsymbol{\beta}\left(\tau^{N}\right)\right) \\
& =V(t, y, \boldsymbol{\beta})+\int_{t}^{\tau^{N}} e^{-\int_{t}^{s} \rho(l) d l} \mathcal{L}^{\mathbf{u}, c} V\left(s, Y^{\mathbf{u}, c}(s), \boldsymbol{\beta}(s)\right) d s+\int_{t}^{\tau^{N}}\{\cdots\} d \mathbf{W}(s) .
\end{aligned}
$$

It is noted that functions $V\left(s, Y^{\mathbf{u}, c}(s), \boldsymbol{\beta}(s)\right), e^{-\int_{t}^{s} \rho(l) d l}$ and all the coefficients and derivatives in $\mathcal{L}^{\mathbf{u}, c}\left[V\left(s, Y^{\mathbf{u}, c}(s), \boldsymbol{\beta}(s)\right)\right]$ are bounded on $s \in\left[0, \tau^{N}\right]$ since $V(t, y, \boldsymbol{\beta}) \in \mathcal{C}^{1,2,2}\left([0, T] \times \mathbf{R} \times \mathbf{R}^{m}\right)$. After applying the Dynkin's formula to (3.42) and taking conditional expectation on both sides, we obtain

$\mathbf{E}_{t}\left[e^{-\int_{t}^{\tau^{N}} \rho(l) d l} V\left(\tau^{N}, Y^{\mathbf{u}, c}\left(\tau^{N}\right), \boldsymbol{\beta}\left(\tau^{N}\right)\right)\right]=V(t, y, \boldsymbol{\beta})+\mathbf{E}_{t}\left[\int_{t}^{\tau^{N}} e^{-\int_{t}^{s} \rho(l) d l} \mathcal{L}^{\mathbf{u}, c} V\left(s, Y^{\mathbf{u}, c}(s), \boldsymbol{\beta}(s)\right) d s\right]$ 
Since $V(t, y, \boldsymbol{\beta})$ satisfies the HJB equation (3.3), for any $(\mathbf{u}, c) \in \mathbf{R}^{m} \times \mathbf{R}$, we have

$$
\mathcal{L}^{\mathbf{u}, c} V(t, y, \boldsymbol{\beta})+U(c) \leq 0
$$

Combining (3.42) and (3.43) results in

$$
V(t, y, \boldsymbol{\beta}) \geq \mathbf{E}_{t}\left[\int_{t}^{\tau^{N}} e^{-\int_{t}^{s} \rho(l) d l} U(c(s)) d s+e^{-\int_{t}^{\tau^{N}} \rho(l) d l} V\left(\tau^{N}, Y^{\mathbf{u}, c}\left(\tau^{N}\right), \boldsymbol{\beta}\left(\tau^{N}\right)\right)\right]
$$

On one hand, it is obvious that

$$
0 \leq \int_{t}^{\tau^{N}} e^{-\int_{t}^{s} \rho(l) d l} e^{-\eta c(s)} d s \uparrow \int_{t}^{T} e^{-\int_{t}^{s} \rho(l) d l} e^{-\eta c(s)} d s<\infty
$$

Applying the monotone convergence theorem results in

$$
\lim _{N \rightarrow \infty} \mathbf{E}_{t}\left[\int_{t}^{\tau^{N}} e^{-\int_{t}^{s} \rho(l) d l} U(c(s)) d s\right]=\mathbf{E}_{t} \int_{t}^{T}\left[e^{-\int_{t}^{s} \rho(l) d l} U(c(s)) d s\right] .
$$

On the other hand, from Lemma 4, we know that the family $\left\{V\left(\tau, Y^{\mathbf{u}, c}(\tau), \boldsymbol{\beta}(\tau)\right\}_{\tau \in \mathcal{K}}\right.$ is uniformly integrable. Moreover, $\rho(t)$ is bounded and $V(t, y, \boldsymbol{\beta}) \in \mathcal{C}^{1,2,2}\left([0, T] \times \mathbf{R} \times \mathbf{R}^{m}\right)$. Now we can exchange the order of limit and expectation and obtain

$$
\lim _{N \rightarrow \infty} \mathbf{E}_{t}\left[e^{-\int_{t}^{\tau^{N}} \rho(l) d l} V\left(\tau^{N}, Y^{\mathbf{u}, c}\left(\tau^{N}\right), \boldsymbol{\beta}\left(\tau^{N}\right)\right)\right]=\mathbf{E}_{t}\left[e^{-\int_{t}^{T} \rho(l) d l} U\left(Y^{\mathbf{u}, c}(T)\right)\right] .
$$

Combining (3.45), (3.47) and (3.48) results in

$$
V(t, y, \boldsymbol{\beta}) \geq \mathbf{E}_{t} \int_{t}^{T}\left[e^{-\int_{t}^{s} \rho(l) d l} U(c(s)) d s+e^{-\int_{t}^{T} \rho(l) d l} U\left(Y^{\mathbf{u}, c}(T)\right)\right]=J(t, y, \boldsymbol{\beta} ; \mathbf{u}(\cdot), c(\cdot)) .
$$

Through replacing $(\mathbf{u}, c)$ with $\left(\mathbf{u}^{*}, c^{*}\right)$, all the inequalities becomes equalities since (3.44) becomes an equality, which leads to

$$
V(t, y, \boldsymbol{\beta})=\mathbf{E}_{t} \int_{t}^{T}\left[e^{-\int_{t}^{s} \rho(l) d l} U\left(c^{*}(s)\right) d s+e^{-\int_{t}^{T} \rho(l) d l} U\left(Y^{\mathbf{u}^{*}, c^{*}}(T)\right)\right]=J\left(t, y, \boldsymbol{\beta} ; \mathbf{u}^{*}(\cdot), c^{*}(\cdot)\right) .
$$

Combining (3.49) and (3.50) leads to the completion of the proof. 


\section{Discussions}

In this section, we discuss the effects of cointegration on the optimal investment and consumption strategies according to the obtained analytical formula in Theorem 1. First of all, our model includes the Merton model (Merton, 1969) as a degenerated case. The optimal strategies in our problem can be decomposed into two parts. The former corresponds to the myopic part; while the latter represents the hedging demand resulted from cointegration. A specific cointegration model is also presented with discussions on how cointegration affects the optimal strategies and improves the investor's optimal expected utility. Finally, some numerical results are provided to support our conclusion that ignoring the information about cointegration results in a utility loss.

\subsection{Degenerated case}

First of all, we illustrate that our model can be considered as an extension to the classic model (Merton, 1969). Assume that the coefficient matrix of cointegration is a zero matrix, i.e. $\mathbf{A} \equiv \mathbf{0}$, and the covariance matrix $\boldsymbol{\Sigma}$ is diagonal with constant entries. We further assume that the risk-free interest rate $r(t)$, the discount rate $\rho(t)$, the drift $\boldsymbol{\mu}(t)$ and the long-time mean level $\boldsymbol{\theta}(t)$ are all constant. Under these assumptions, the dynamics of stock price (2.6) degenerates to a geometric Brownian motion and the corresponding optimization problem degenerates to the classic Merton problem.

Let us quickly demonstrate the degeneracy. According to Lemma 3, we have

$$
\left\{\begin{array}{l}
K_{1}(t)=\frac{r-\rho}{r-1+e^{r(T-t)}}\left[\frac{e^{r(T-t)}-1}{r}+(r-1)(T-t)\right]-\ln h(t), \\
\mathbf{K}_{2}(t)=\mathbf{0}_{m \times 1}, \\
\mathbf{K}_{3}(t)=-\frac{1}{r-1+e^{r(T-t)}}\left[\frac{e^{r(T-t)}-1}{r}+(r-1)(T-t)\right] \mathbf{\Sigma}^{-1}
\end{array}\right.
$$

Consequently, the optimal investment and consumption strategies become

$$
\left\{\begin{array}{l}
\mathbf{u}_{M}^{*}(t, \boldsymbol{\beta})=\frac{1}{\eta h(t)} \boldsymbol{\Sigma}^{-1} \boldsymbol{\beta}, \\
c_{M}^{*}(t, y, \boldsymbol{\beta})=h(t) y-\frac{1}{\eta} \frac{r-\rho-0.5 \boldsymbol{\beta}^{\top} \boldsymbol{\Sigma}^{-1} \boldsymbol{\beta}}{r-1+e^{r(T-t)}}\left[\frac{e^{r(T-t)}-1}{r}+(r-1)(T-t)\right],
\end{array}\right.
$$

which are exactly the same as the results in the Merton's case (Chen et al., 2012). In the 
degenerated case, the optimal investment amount $\mathbf{u}_{M}^{*}$ is proportional to the risk premium $\boldsymbol{\beta}$, reciprocal of the coefficient of the absolute risk aversion $\eta$ but independent of the investor's initial wealth. If the risk premium is positive, the investor should long stocks; whereas the investor should otherwise short the stocks. Moreover, the optimal consumption rate $c_{M}^{*}$ is a linear function of wealth $y$.

\section{$4.2 \quad$ Non-degenerated case}

In order to explore how cointegration affects optimal investment and consumption strategies, the optimal strategies in (3.17) are decomposed into two parts:

$$
\left\{\begin{array}{l}
\mathbf{u}^{*}(t, \boldsymbol{\beta})=\mathbf{u}_{M}^{*}(t, \boldsymbol{\beta})+\mathbf{u}_{A}^{*}(t, \boldsymbol{\beta}), \\
c^{*}(t, y, \boldsymbol{\beta})=c_{M}^{*}(t, y, \boldsymbol{\beta})+c_{A}^{*}(t, \boldsymbol{\beta}),
\end{array}\right.
$$

where

$$
\left\{\begin{array}{l}
\mathbf{u}_{A}^{*}(t, \boldsymbol{\beta})=-\frac{1}{\eta h(t)} \mathbf{A}^{\top}\left[\mathbf{K}_{3}(t) \boldsymbol{\beta}+\mathbf{K}_{2}(t)\right], \\
c_{A}^{*}(t, \boldsymbol{\beta})=-\frac{1}{\eta}\left\{\mathbf{K}_{2}(t)^{\top} \boldsymbol{\beta}+\int_{t}^{T} e^{-\int_{t}^{s} h(l) d l}\left[\mathbf{K}_{2}(s)^{\top} \boldsymbol{\Theta}+\frac{1}{2} \operatorname{tr}\left(\mathbf{K}_{3}(s) \mathbf{A} \boldsymbol{\Sigma} \mathbf{A}^{\top}\right)\right] d s\right\} .
\end{array}\right.
$$

Both $\mathbf{u}_{M}^{*}$ and $c_{M}^{*}$ represent the myopic part (i.e. the Merton component) when there is no cointegration. Both $\mathbf{u}_{A}^{*}$ and $c_{A}^{*}$ are the adjustment terms (or the hedging demand) resulted from the cointegration. One of the main contributions of this paper is that both of these adjustment terms are in closed form, and they can thus be analyzed analytically to demonstrate the static analysis.

A specific cointegration model is presented to further study the effects of cointegration. For simplicity, let us consider a financial market with one risk-free asset and two risky assets. The logprices of risky assets are $\mathbf{X}(t)=\left(X_{1}(t), X_{2}(t)\right)^{\top}=\left(\ln S_{1}(t), \ln S_{1}(t)\right)^{\top}$ and the other parameters are assumed to be constant as

$$
\left.\boldsymbol{\theta}=\left(\theta_{1}, \theta_{2}\right)^{\top}, \quad \boldsymbol{\sigma}=\operatorname{diag}\left\{\left(\sigma_{1}, \sigma_{2}\right)^{\top}\right)\right\}
$$

The coefficient matrix of cointegration A plays a critical role in the cointegration model. It can 
be taken of different forms to represent various cointegration patterns. In practice, the coefficient matrix of cointegration is determined by the calibrations based on historical market data. A particular cointegration pattern is observed by Chiu and Wong (2011) and the corresponding coefficient matrix is also extracted accordingly.

To illustrate a simple example, following Chiu and Wong (2013b), we take the coefficient matrix of cointegration as the following form

$$
\mathbf{A}=\frac{\epsilon}{2}\left(\begin{array}{ll}
1 & 1 \\
1 & 1
\end{array}\right), \quad \epsilon>0
$$

so that the cointegrating factor $z(t)=X_{1}(t)+X_{2}(t)=\ln S_{1}(t)+\ln S_{1}(t)$ is a stationary process satisfying

$$
d z(t)=\left[\left(\theta_{1}+\theta_{2}\right)-\epsilon z(t)\right] d t+\sigma_{1} d W_{1}(t)+\sigma_{2} d W_{2}(t)
$$

where $\epsilon$ represents the mean-reverting speed of cointegrating factor. In other words, the sum of log-returns of two risky assets forms a stationary process, which is very a common pattern of cointegration that is observed in the practice of pairs trading (Lee and Papanicolaou, 2016). The mean-reverting speed $\epsilon$ plays an important role in a cointegration model. The larger $\epsilon$, the more pronounced the cointegration effect. In the following, most of the discussions would be carried out when this key parameter varies.

By applying Lemma 3, we have

$$
\mathbf{K}_{3}(t)=k_{3}(t) \operatorname{diag}\left\{\left(\sigma_{1}^{-2}, \sigma_{2}^{-2}\right)^{\top}\right\}, \quad \mathbf{K}_{2}(t)=k_{2}(t) \frac{\epsilon\left(\sigma_{1}^{2}+\sigma_{2}^{2}-4 r\right)}{4}(1,1)^{\top},
$$

where

$$
k_{2}(t)=-\frac{1}{r-1+e^{r(T-t)}}\left[\frac{e^{r(T-t)}-1}{r^{2}}+\frac{(r-1)(T-t)^{2}}{2}-\frac{T-t}{r}\right]<0,
$$

and

$$
k_{3}(t)=-\frac{1}{r-1+e^{r(T-t)}}\left[\frac{e^{r(T-t)}-1}{r}+(r-1)(T-t)\right]<0 .
$$

As a direct application of Theorem 1, we arrive at the following proposition.

Proposition 1. When the coefficient matrix of cointegration in the cointegrated model is taken 
as (4.5), the optimal expected utility under the cointegration model is expressed as

$$
V(t, y, \boldsymbol{\beta})=V_{M}(t, y, \boldsymbol{\beta}) \times R(t, \boldsymbol{\beta})
$$

where $V_{M}$ represents the optimal expected utility in the Merton's case and $R$ denotes the amplification factor of the cointegration. The analytical expressions for $V_{M}$ and $R$ are

$$
\left\{\begin{array}{l}
V_{M}(t, y, \boldsymbol{\beta})=-\frac{1}{\eta} \exp \left\{-\eta h(t) y+\frac{r-\rho-\frac{1}{2}\left(\frac{\beta_{1}^{2}}{\sigma_{2}^{2}}+\frac{\beta_{2}^{2}}{\sigma_{2}^{2}}\right)}{r-1+e^{r(T-t)}}\left[\frac{e^{r(T-t)}-1}{r}+(r-1)(T-t)\right]\right\}, \\
R(t, \boldsymbol{\beta})=\exp \left\{\frac{\left(\beta_{1}+\beta_{2}\right)\left(\sigma_{1}^{2}+\sigma_{2}^{2}-4 r\right)}{4} k_{2}(t) \epsilon+\frac{f_{1} g_{1}(t)+f_{2} g_{2}(t)}{r-1+e^{r(T-t)}} \epsilon^{2}\right\},
\end{array}\right.
$$

with functions $g_{1}(t)=-\frac{e^{r}(T-t)-1}{r^{3}}+\frac{T-t}{r^{2}}-\frac{(r-1)(T-t)^{3}}{6}+\frac{(T-t)^{2}}{2 r}<0, g_{2}(t)=-\frac{e^{r}(T-t)-1}{r^{2}}+\frac{T-t}{r}-$ $\frac{(r-1)(T-t)^{2}}{2}<0$ and constants $f_{1}=\frac{\left(\sigma_{1}^{2}+\sigma_{2}^{2}-4 r\right)^{2}}{8}, f_{2}=\frac{1}{8}\left(2+\frac{\sigma_{1}^{2}}{\sigma_{2}^{2}}+\frac{\sigma_{2}^{2}}{\sigma_{1}^{2}}\right)$. In addition, the optimal investment and consumption strategies are given in (4.3) with

$$
\left\{\begin{array}{l}
\mathbf{u}_{M}^{*}(t, \boldsymbol{\beta})=\frac{1}{\eta h(t)}\left(\frac{\beta_{1}}{\sigma_{1}^{2}}, \frac{\beta_{1}}{\sigma_{2}^{2}}\right)^{\top} \\
\mathbf{u}_{A}^{*}(t, \boldsymbol{\beta})=-\frac{1}{8 \eta h(t)}\left[4 k_{3}(t)\left(\frac{\beta_{1}}{\sigma_{1}^{2}}+\frac{\beta_{2}}{\sigma_{2}^{2}}\right) \epsilon+k_{2}(t)\left(\sigma_{1}^{2}+\sigma_{2}^{2}-4 r\right) \epsilon^{2}\right](1,1)^{\top} \\
c_{M}^{*}(t, y, \boldsymbol{\beta})=h(t) y-\frac{1}{\eta} \frac{r-\rho-0.5 \beta^{T} \Sigma^{-1} \beta}{r-1+e^{r(T-t)}}\left[\frac{e^{r(T-t)}-1}{r}+(r-1)(T-t)\right] \\
c_{A}^{*}(t, \boldsymbol{\beta})=-\frac{1}{\eta}\left[\frac{\left(\sigma_{1}^{2}+\sigma_{2}^{2}-4 r\right)\left(\beta_{1}+\beta_{2}\right) k_{2}(t)}{4} \epsilon+\frac{f_{1} g_{1}(t)+f_{2} g_{2}(t)}{r-1+e^{r(T-t)}} \epsilon^{2}\right]
\end{array}\right.
$$

It has to be pointed out that a key question in this paper is how the cointegration affects the optimal investment and consumption strategies. Now such a question can be clearly and quantitatively answered, based on the obtained analytical formula in (4.3) and (4.11)-(4.13). Obviously, the cointegration affects not only the optimal expected utility but also the optimal strategies. From (4.11), whether or not the cointegration enhances the optimal expected utility depends on the value of the amplification factor $R$. If $R<1$, the optimal expected utility is indeed improved with respect to the classic Merton's case, while the optimal expected utility has worsen off when $R>1^{2}$. On the other hand, how the cointegration affects the optimal strategies can be understood from (4.3) and (4.13). The adjustment term $\mathbf{u}_{A}^{*}$ would guide an investor, who takes advantage of the cointegration information, to adjust his allocation accordingly, while $c_{A}^{*}$ tells him to adjust his consumption rate accordingly as well. If $c_{A}^{*}$ is positive, it implies

\footnotetext{
${ }^{2}$ It should be pointed out that $V_{M}(t, y, \boldsymbol{\beta})$ is always negative.
} 
that the cointegration increases the consumption rate over the classic Merton's case, while the cointegration decreases the consumption rate if $c_{A}^{*}$ is negative. Specifically, a positive $c_{A}^{*}$ may reduce the likelihood of negative consumption when $c_{M}^{*}$ is negative.

Now, we study how the optimal expected utility varies with different values of the meanreverting speed $\epsilon$. In (4.11), $V_{M}$ corresponds to the optimal expected utility in the classic Merton problem, which is independent of $\epsilon$, as expected. After some simple calculations, we find that functions $g_{1}(t), g_{2}(t)$ and $k_{2}(t)$ are all negative. Consequently, the monotonicity of $V(t, y, \boldsymbol{\beta})$ is shown in Table $1^{3}$ :

\begin{tabular}{|c|c|c|}
\hline & $\sigma_{1}^{2}+\sigma_{2}^{2}-4 r>0$ & $\sigma_{1}^{2}+\sigma_{2}^{2}-4 r<0$ \\
\hline$\beta_{1}+\beta_{2}>0$ & $V \uparrow \epsilon$ & $V \uparrow \epsilon$ on $\left[0, \epsilon_{1}^{*}\right], V \downarrow \epsilon$ on $\left[\epsilon_{1}^{*}, \infty\right)$ \\
\hline$\beta_{1}+\beta_{2}<0$ & $V \uparrow \epsilon$ on $\left[0, \epsilon_{1}^{*}\right], V \downarrow \epsilon$ on $\left[\epsilon_{1}^{*}, \infty\right)$ & $V \uparrow \epsilon$ \\
\hline
\end{tabular}

Table 1: Monotonicity of the value function $V(t, y, \boldsymbol{\beta})$ with respect to $\epsilon$.

The Merton components in optimal investment and consumption strategies, $\mathbf{u}_{M}^{*}$ and $c_{M}^{*}$, are both independent of mean-reverting speed $\epsilon$ as they should. The cointegration effect is shown in the adjustment terms $\mathbf{u}_{A}^{*}$ and $c_{A}^{*}$. The cointegration effect disappears if $\epsilon=0$. In addition, the monotonicity of adjustment terms with respect to $\epsilon$ can be summarized in Table 2 and Table 3 :

\begin{tabular}{|c|c|c|}
\hline & $\sigma_{1}^{2}+\sigma_{2}^{2}-4 r>0$ & $\sigma_{1}^{2}+\sigma_{2}^{2}-4 r<0$ \\
\hline$\frac{\beta_{1}}{\sigma_{1}^{2}}+\frac{\beta_{2}}{\sigma_{2}^{2}}>0$ & $\mathbf{u}_{A}^{*} \uparrow \epsilon$ & $\mathbf{u}_{A}^{*} \uparrow \epsilon$ on $\left[0, \epsilon_{2}^{*}\right], \mathbf{u}_{A}^{*} \downarrow \epsilon$ on $\left[\epsilon_{2}^{*}, \infty\right)$ \\
\hline$\frac{\beta_{1}}{\sigma_{1}^{2}}+\frac{\beta_{2}}{\sigma_{2}^{2}}<0$ & $\mathbf{u}_{A}^{*} \downarrow \epsilon$ on $\left[0, \epsilon_{2}^{*}\right], \mathbf{u}_{A}^{*} \uparrow \epsilon$ on $\left[\epsilon_{2}^{*}, \infty\right)$ & $\mathbf{u}_{A}^{*} \downarrow \epsilon$ \\
\hline
\end{tabular}

Table 2: Monotonicity of the hedging demand $\mathbf{u}_{A}^{*}$ with respect to $\epsilon$.

\begin{tabular}{|c|c|c|}
\hline & $\sigma_{1}^{2}+\sigma_{2}^{2}-4 r>0$ & $\sigma_{1}^{2}+\sigma_{2}^{2}-4 r<0$ \\
\hline$\beta_{1}+\beta_{2}>0$ & $c_{A}^{*} \uparrow \epsilon$ & $c_{A}^{*} \uparrow \epsilon$ on $\left[0, \epsilon_{1}^{*}\right], c_{A}^{*} \downarrow \epsilon$ on $\left[\epsilon_{1}^{*}, \infty\right)$ \\
\hline$\beta_{1}+\beta_{2}<0$ & $c_{A}^{*} \uparrow \epsilon$ on $\left[0, \epsilon_{1}^{*}\right], c_{A}^{*} \downarrow \epsilon$ on $\left[\epsilon_{1}^{*}, \infty\right)$ & $c_{A}^{*} \uparrow \epsilon$ \\
\hline
\end{tabular}

Table 3: Monotonicity of adjustment term $c_{A}^{*}$ with respect to $\epsilon$.

From Tables 1-3, we know that the monotonicity depends on the model parameters. To demonstrate the behavior of optimal investment and consumption strategies and the corresponding value function specifically, we adopt the parameter values for the cointegration model used in

\footnotetext{
${ }^{3}$ In Tables 1,2 and $3, \epsilon_{1}^{*}=\frac{\left(\beta_{1}+\beta_{2}\right)\left(\sigma_{2}^{2}+\sigma_{2}^{2}-4 r\right) k_{2}(t)}{\left.8\left[f_{1} g_{1}(t)\right)+f_{2} g_{2}(t)\right]}$ and $\epsilon_{2}^{*}=-\frac{2 k_{3}(t)}{k_{2}(t)\left(\sigma_{1}^{2}+\sigma_{2}^{2}-4 r\right)}\left(\frac{\beta_{1}}{\sigma_{1}^{2}}+\frac{\beta_{2}}{\sigma_{2}^{2}}\right)$.
} 
Chiu and Wong (2011) and Shen and Siu (2017) as follow:

$$
\begin{array}{r}
r=0.03, \rho=0.04, \boldsymbol{\sigma}=\operatorname{diag}\left\{(0.3,0.3)^{\top}\right\}, T=1, \eta=1, \\
\boldsymbol{\beta}_{0}=(0.0234,0.0334)^{\top}, y_{0}=10, \boldsymbol{\theta}=(0.1,0.2)^{\top}, x_{0}=(\ln 1, \ln 2)^{\top} .
\end{array}
$$

Remark 6. The chosen parameters in (4.14) satisfy $\sigma_{1}^{2}+\sigma_{2}^{2}-4 r>0$ and $\beta_{1}+\beta_{2}>0$. Therefore, the value function $V(t, y, \boldsymbol{\beta})$ and optimal strategies $\mathbf{u}^{*}$ and $c^{*}$ are all increasing as the meanreverting speed goes up as demonstrated in Tables 1-3.

To measure the improvement of utility resulted from cointegration, a ratio is defined as

$$
\text { Relative Ratio }=\frac{\mathbf{J}\left(0, y_{0}, \boldsymbol{\beta} ;\left(\mathbf{u}^{*}(\cdot), c^{*}(\cdot)\right)\right)-V_{M}\left(0, y_{0}\right)}{\left|V_{M}\left(0, y_{0}\right)\right|}=\frac{V\left(0, y_{0}, \boldsymbol{\beta}_{0}\right)-V_{M}\left(0, y_{0}\right)}{\left|V_{M}\left(0, y_{0}\right)\right|} \times 100 \%,
$$

which is plotted in Figure 1 with different values of $\epsilon$.

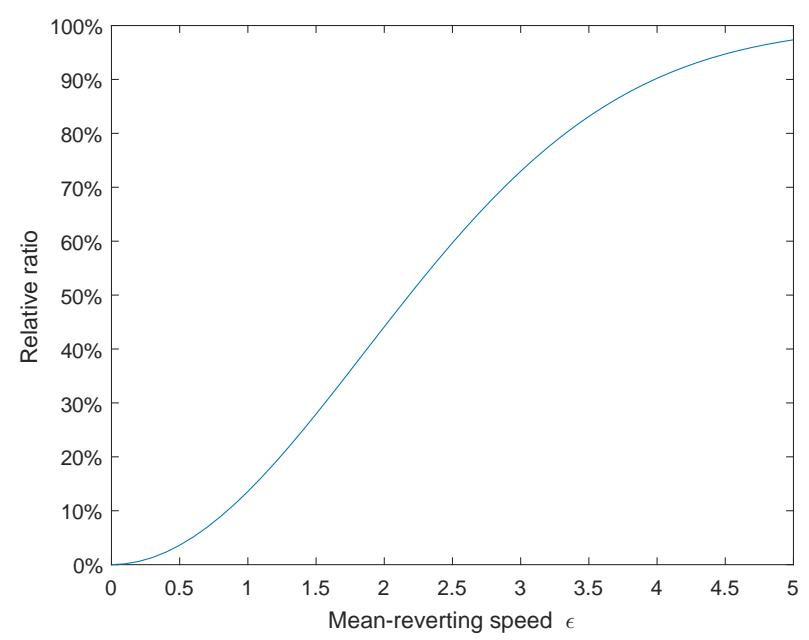

Figure 1: Relative ratio of the optimal expected utility with different values of $\epsilon$.

From Figure 1, the relative ratio is non-negative, which implies that the cointegration increases the optimal expected utility over the classic Merton case. As the mean-reverting speed goes up from 0 to 5, the relative ratio increases from 0 to $96 \%$. In other words, the larger the meanreverting speed, the more pronounced the cointegration boosts the expected utility. Another observation is that the relative ratio is increasing nonlinearly with respect to the mean-reversion speed $\epsilon$. From Figure 1, the ratio increases very quickly at the very beginning, but the rate of 
increment slows down later as the value of $\epsilon$ exceeds a certain value (about 2.5). From a financial point of view, an investor, who takes advantage of the cointegration information, could improve his optimal expected utility, while an investor, who ignores the information about cointegration, would suffer a utility loss. Consequently, it is very important for investors to observe the cointegration phenomena in financial market and then make full use of them in order to maximize their expected utility.

Under the parameters in (4.14), the optimal investment and consumption strategies are plotted against different levels of mean-reverting speed in Figure 2.

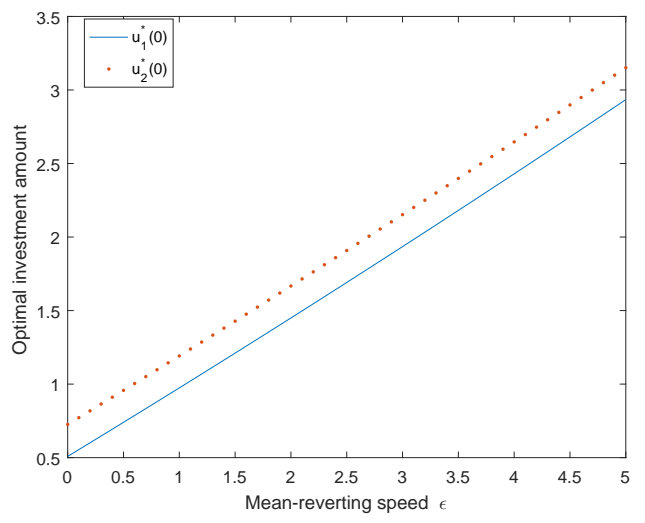

(a) Optimal investment amount at time $t=0$.

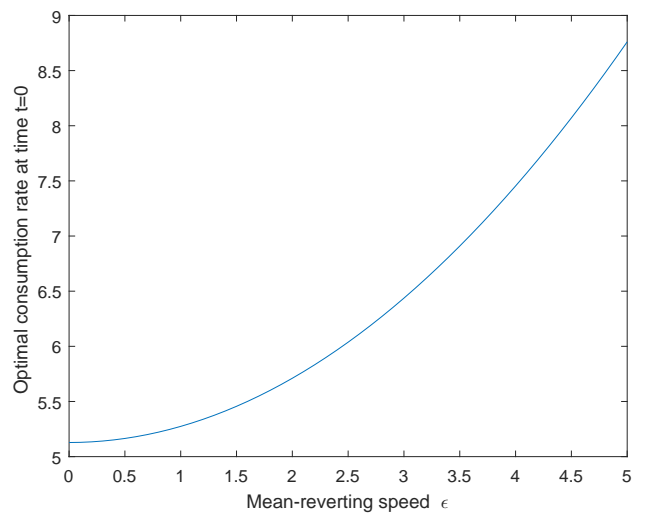

(b) Optimal consumption rate at time $t=0$.

Figure 2: Optimal investment amount and consumption rate.

From Figure 2, both the investment amount and consumption rate at time $t=0$ are increasing functions of the mean-reverting speed $\epsilon$ as stated in Remark 6. The more pronounced the cointegration effect, the more investment on stocks and larger consumption rate. The difference between investment on Stock 1 and Stock 2 is due to the difference in risk premium $\beta_{1}$ and $\beta_{2}$. From the analytical expression for $\mathbf{u}_{A}^{*}$ in (4.13), we can conclude that the hedging demand caused by cointegration is the same for these two stocks, and also is independent of the risk premium. A potential reason is that Stock 1 and Stock 2 have the same weight in the cointegrating factor $z(t)=X_{1}(t)+X_{2}(t)$.

We now examine the sign of the hedging demand $\mathbf{u}_{A}^{*}$ and the adjustment term $c_{A}^{*}$ for consumption strategies. All the parameters are set as (4.14), except the time horizon $T$. The hedging demand $\mathbf{u}_{A}^{*}$ and the adjustment term $c_{A}^{*}$ depicted in Figure 3 are all positive, which implies that 
the investor should invest and consume more compared with the Merton case. In addition, as demonstrated in Remark 6, both of them increases as the mean-reverting speed $\epsilon$ becomes large, indicating that the more pronounced cointegration effect, the more the investor would increase his investment amount and consumption. Moreover, the horizon effect of the cointegration is also

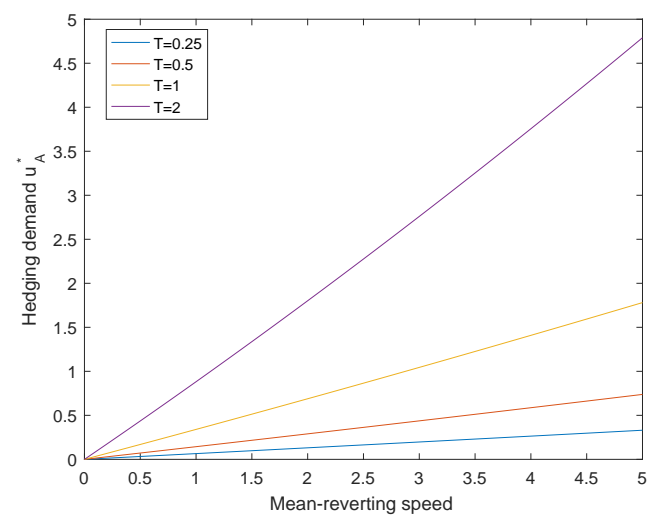

(a) Horizon effect for the hedging demand $\mathbf{u}_{A}^{*}$.

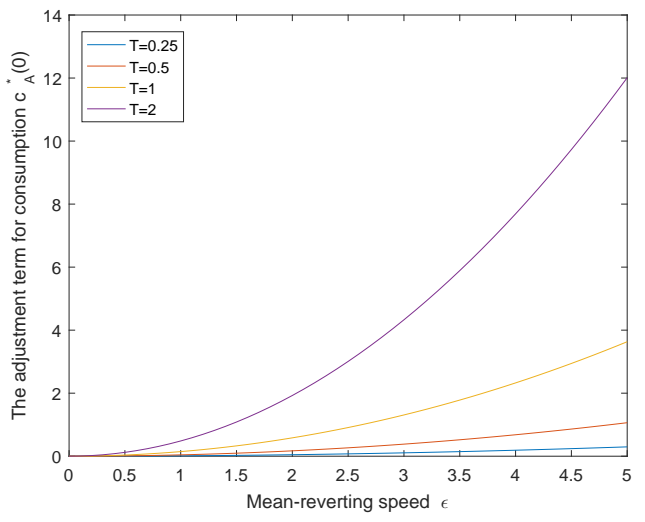

(b) Horizon effect for the adjustment term of consumption rate.

Figure 3: Horizon effect.

shown in Figure 3. As expected, the longer time horizon, the more pronounced the cointegration effect, because the investor can benefit from the cointegration information for a longer time.

Finally, we point out that all the discussions in this subsection are carried out under the parameter setting (4.14). For other sets of parameters, as a direct benefit of newly derived analytical expressions for optimal investment and consumption strategies, similar discussions could also be carried out.

\section{Conclusions}

This paper demonstrates that, under the continuous-time cointegration model, the optimal investment and consumption strategies for an investor with exponential utility can be expressed in closed form via solving the corresponding HJB equation analytically. A verification theorem is also established to demonstrate that the solution to the HJB equation is indeed the solution to the original problem under an exponential integrability condition. Moreover, a simple and sufficient condition is proposed to ensure that such an exponential integrability condition is satisfied. 
In this paper, the optimal investment and consumption strategies are decomposed into two parts: the myopic part and the hedging demand caused by cointegration. When the cointegration effect disappears, our model degenerates to the classic Merton case. A specific cointegration model is also provided as an example to demonstrate how the cointegration affects the optimal strategies. Under the chosen parameters, cointegration improves the optimal expected utility more and more as it becomes more and more pronounced. In other words, an investor who ignores the cointegration information suffers a significant utility loss. Finally, the horizon effect are also provided, i.e. the longer horizon, the more the investor benefits from the cointegration information.

\section{Acknowledgment}

The research conducted and reported in this paper is part of an $\mathrm{ARC}($ Australian Research Council)-funded three-year project, for which the authors gratefully acknowledge the financial support from the ARC under the ARC(DP) funding scheme (DP140102076, DP170101227). The authors would also like to thank two anonymous referees for their valuable comments and suggestions, which have led to a substantial improvement of the readability of the paper.

\section{Appendix A Calculation of higher moments of multivariate nor- mal distribution}

To calculate higher moments of multivariate normal distribution, we introduce spherical coordinate system as follows:

$$
\begin{aligned}
x_{1} & =r \cos \left(\phi_{1}\right) \\
x_{2} & =r \sin \left(\phi_{1}\right) \cos \left(\phi_{2}\right) \\
& \cdots \\
x_{m-1} & =r \sin \left(\phi_{1}\right) \cdots \sin \left(\phi_{m-2}\right) \cos \left(\phi_{m-1}\right) \\
x_{m} & =r \sin \left(\phi_{1}\right) \cdots \sin \left(\phi_{m-2}\right) \sin \left(\phi_{m-1}\right) .
\end{aligned}
$$


Then, we calculate as follows:

$$
\begin{aligned}
& \mathbf{E}\|\mathbf{Q}\|_{m}^{2 n} \\
& =\frac{1}{(2 \pi)^{\frac{m}{2}}} \int_{\mathbf{R}^{m}}\left(x_{1}^{2}+x_{2}^{2}+\cdots+x_{m}^{2}\right)^{n} e^{-\frac{x_{1}^{2}+x_{2}^{2}++x_{m}^{2}}{2}} d x_{1} d x_{2} \cdots d x_{m} \\
& =\frac{1}{(2 \pi)^{\frac{m}{2}}} \int_{0}^{2 \pi} d \phi_{m-1} \int_{0}^{\pi} \cdots \int_{0}^{\pi} \sin ^{m-2}\left(\phi_{1}\right) \sin ^{m-3}\left(\phi_{2}\right) \cdots \sin \left(\phi_{m-2}\right) d \phi_{1} \cdots d \phi_{m-2} \int_{0}^{\infty} r^{m-1} r^{2 n} e^{-\frac{r^{2}}{2}} d r \\
& =2^{n} \pi^{1-\frac{m}{2}} \Upsilon \int_{0}^{\infty} u^{n+\frac{m}{2}-1} e^{-u} d u \\
& =2^{n} \pi^{1-\frac{m}{2}} \Upsilon \Gamma\left(n+\frac{m}{2}\right),
\end{aligned}
$$

where $\Upsilon=\int_{0}^{\pi} \cdots \int_{0}^{\pi} \sin ^{m-2}\left(\phi_{1}\right) \sin ^{m-3}\left(\phi_{2}\right) \cdots \sin \left(\phi_{m-2}\right) d \phi_{1} \cdots d \phi_{m-2}$ and $\Gamma(n)=\int_{0}^{\infty} x^{n-1} e^{-x} d x$.

Furthermore, we have identity $\Gamma(n+1)=n \Gamma(n)$.

\section{References}

Alexander, C. (1999). Optimal hedging using cointegration. Philosophical Transactions of the Royal Society of London A: Mathematical, Physical and Engineering Sciences, 357(1758):20392058.

Baillie, R. T. and Bollerslev, T. (1989). Common stochastic trends in a system of exchange rates. Journal of Finance, 44(1):167-181.

Benth, F. E. and Karlsen, K. H. (2005). A note on merton's portfolio selection problem for the schwartz mean-reversion model. Stochastic Analysis and Applications, 23(4):687-704.

Brennan, M. J., Schwartz, E. S., and Lagnado, R. (1997). Strategic asset allocation. Journal of Economic Dynamics and Control, 21(8):1377-1403.

Campbell, J. Y. (1987). Stock return and the term structure. Journal of Financial Economics, 18:373-399.

Campbell, J. Y. and Shiller, R. J. (1988). The dividend-price ratio and expectations of future dividends and discount factors. Review of Financial Studies, 1(3):195-228.

Cerchi, M. and Havenner, A. (1988). Cointegration and stock prices: The random walk on wall street revisited. Journal of Economic Dynamics and Control, 12(2-3):333-346.

Chen, Y., Dai, M., and Zhao, K. (2012). Finite horizon optimal investment and consumption with cara utility and proportional transaction costs. In Stochastic Analysis and Applications to Finance: Essays in Honour of Jia-an Yan, pages 39-54. World Scientific.

Chiu, M. C. and Wong, H. Y. (2011). Mean-variance portfolio selection of cointegrated assets. Journal of Economic Dynamics and Control, 35(8):1369-1385.

Chiu, M. C. and Wong, H. Y. (2012). Mean-variance asset-liability management: Cointegrated assets and insurance liability. European Journal of Operational Research, 223(3):785-793. 
Chiu, M. C. and Wong, H. Y. (2013a). Mean-variance principle of managing cointegrated risky assets and random liabilities. Operations Research Letters, 41(1):98-106.

Chiu, M. C. and Wong, H. Y. (2013b). Optimal investment for an insurer with cointegrated assets: Crra utility. Insurance: Mathematics and Economics, 52(1):52-64.

Duan, J.-C. and Pliska, S. R. (2004). Option valuation with co-integrated asset prices. Journal of Economic Dynamics and Control, 28(4):727-754.

Engle, R. F. and Granger, C. W. (1987). Co-integration and error correction: representation, estimation, and testing. Econometrica, 55(2):251-276.

Fama, E. F. and French, K. R. (1988). Permanent and temporary components of stock prices. Journal of Political Economy, 96(2):246-273.

Fleming, W. H. and Soner, H. M. (2006). Controlled Markov processes and viscosity solutions, volume 25. Springer Science \& Business Media.

Granger, C. W. (1981). Some properties of time series data and their use in econometric model specification. Journal of econometrics, 16(1):121-130.

Kim, T. S. and Omberg, E. (1996). Dynamic nonmyopic portfolio behavior. Review of Financial Studies, 9(1):141-161.

Korn, R. and Kraft, H. (2004). On the stability of continuous-time portfolio problems with stochastic opportunity set. Mathematical Finance, 14(3):403-414.

Lee, S. and Papanicolaou, A. (2016). Pairs trading of two assets with uncertainty in cointegration's level of mean reversion. International Journal of Theoretical and Applied Finance, 19(08):1650054.

Lim, T., Quenez, M.-C., et al. (2011). Exponential utility maximization in an incomplete market with defaults. Electronic Journal of Probability, 16:1434-1464.

Liu, J. (2007). Portfolio selection in stochastic environments. Review of Financial Studies, 20(1):1-39.

Markowitz, H. (1952). Portfolio selection. Journal of Finance, 7(1):77-91.

Merton, R. C. (1969). Lifetime portfolio selection under uncertainty: The continuous-time case. Review of Economics and Statistics, 51(3):247-257.

Merton, R. C. (1971). Optimum consumption and portfolio rules in a continuous-time model. Journal of Economic Theory, 3(4):373-413.

Mudchanatongsuk, S., Primbs, J. A., and Wong, W. (2008). Optimal pairs trading: A stochastic control approach. In American Control Conference, 2008, pages 1035-1039. IEEE.

Øksendal, B. (2003). Stochastic differential equations: An introduction with applications. Springer, Berlin.

Samuelson, P. A. (1969). Lifetime portfolio selection by dynamic stochastic programming. Review of Economics and Statistics, 51(3):239-246.

Serletis, A. (1994). A cointegration analysis of petroleum futures prices. Energy Economics, 16(2):93-97.

Shen, Y. and Siu, T. K. (2017). Optimal investment and consumption in a continuous-time co-integration model. IMA Journal of Management Mathematics, 28(4):501-530. 
Taylor, M. P. and Tonks, I. (1989). The internationalisation of stock markets and the abolition of uk exchange control. Review of Economics and Statistics, 71(2):332-336.

Tourin, A. and Yan, R. (2013). Dynamic pairs trading using the stochastic control approach. Journal of Economic Dynamics and Control, 37(10):1972-1981.

Wachter, J. A. (2002). Portfolio and consumption decisions under mean-reverting returns: An exact solution for complete markets. Journal of Financial and Quantitative Analysis, 37(01):6391.

Xia, Y. (2001). Learning about predictability: The effects of parameter uncertainty on dynamic asset allocation. Journal of Finance, 56(1):205-246.

Yong, J. and Zhou, X. Y. (1999). Stochastic controls: Hamiltonian systems and HJB equations, volume 43. Springer Science \& Business Media.

Zhu, S.-P. and Ma, G. (2018). An analytical solution for the HJB equation arising from the merton problem. International Journal of Financial Engineering, 5(01):1850008. 